\title{
Observer-Based Event-Triggered Control for Switched Systems with Time-Varying Delay and Norm-Bounded Disturbance
}

\author{
Guoqi Ma, Linlin Qin, Xinghua Liu, and Gang Wu \\ Department of Automation, School of Information Science and Technology, University of Science and Technology of China, \\ Anhui 230027, China \\ Correspondence should be addressed to Guoqi Ma; maguoqi@mail.ustc.edu.cn, Linlin Qin; qinll@ustc.edu.cn, \\ and Xinghua Liu; salxh@mail.ustc.edu.cn
}

Received 31 August 2015; Accepted 2 March 2016

Academic Editor: Naohisa Otsuka

Copyright (C) 2016 Guoqi Ma et al. This is an open access article distributed under the Creative Commons Attribution License, which permits unrestricted use, distribution, and reproduction in any medium, provided the original work is properly cited.

\begin{abstract}
This paper is concerned with the problem of observed-based event-triggered control for switched linear systems with time-varying delay and exogenous disturbance. First by employing a state observer, an observer-based event-triggered controller is designed to guarantee the finite-time boundedness and finite-time stabilization of the resulting dynamic augmented closed-loop system. Then based on the Lyapunov-like function method and the average dwell time technique, some sufficient conditions are given to ensure the finite-time boundedness and finite-time stabilization, respectively. Furthermore, the lower bound of the minimum interevent interval is proved to be positive, which thus excludes the Zeno behavior of sampling. A numerical example is finally exploited to verify the effectiveness and potential of the achieved control scheme.
\end{abstract}

\section{Introduction}

Broadly speaking, hybrid systems are such a class of systems where continuous-time dynamics and discrete-time dynamics interact. Switched systems, consisting of a finite number of subsystems described by differential or difference equations and a logical rule orchestrating the switching order among these subsystems, can be regarded as a special category of hybrid systems. In recent decades, numerous efforts have been devoted to the study of switched systems due to their intrinsic characteristic and their practical applications in a wide range of areas, for example, power electronics [1], networked control systems [2], robot control systems [3], air traffic control systems [4], and modern agriculture systems [5], to list a few. In addition, along the line of theoretical research, a great deal of valuable results on switched systems have been presented, such as stability and stabilization [614], controllability and reachability [15], and observability $[16,17]$. In general, the stability and stabilization problems are the principal concerns for switched systems. Currently, most of the existing literatures on stability and stabilization of switched systems are focused on Lyapunov asymptotic stability, which is defined over an infinite time interval. Nevertheless, in practice, one may be only interested in a bound of system trajectories over a fixed short time interval, as there may exist such a case that a system is Lyapunov stable but completely of no practical use if it possesses undesirable transient performances, such as the systems with saturation elements $[18,19]$. In order to study the transient performances of a system, the concept of finite-time stability was proposed in [20]. To be specific, a system is said to be finite-time stable if, given a bound on the initial state condition, the system state trajectories stay within a prescribed range during a fixed time interval. Hence, the finite-time stability is more practically meaningful compared with Lyapunov asymptotic stability. For more related results on finite-time stability, interested readers can be referred to $[6,14,21-23]$ and references cited therein. Moreover, time-delay is a common phenomenon arising in various practical applications, for example, networked control systems, chemical engineering systems, and power systems [24-28]. And time delays are the inherent characteristics of a large number of physical plants and the 
big sources of instability and poor performances for switched systems [29] as well. Therefore, it is nontrivial to investigate the control problem for switched systems with time delays.

On the other hand, in quite a lot of modern industrial control applications, in order to run real-time operating systems, the controllers are usually implemented on digital platforms which are furnished with microprocessors [30]. In such an implementation, the control task comprises sampling the plant outputs, computing and implementing new control signals. Traditionally, the control task is executed periodically on the basis of the well-developed sampled-data system theory from an analysis and design point of view [31, 32]. Nonetheless, it is well worth pointing out that the aforementioned control strategy is conservative from a resource utilization point of view: that is, sampling at a constant rate regardless of whether it is really necessary or not will result in a waste of communication resource when no disturbances are influencing the system and the system is approaching its desired equilibrium [33]. To address the problems arising from the periodic control scenario, an alternative to sampleddata control paradigm, event-triggered control (ETC), also called event-based control or event-driven control in the literatures, has been proposed; see, for example, [34-36]. In the event-triggered control framework, the control task will not get updated until an external event occurs, generated in light of some prescribed event-triggered mechanism, rather than according to the elapse of a certain fixed time period as utilized in the conventional periodic sampled-data control strategy. Consequently, the number of control task executions and the communication frequency between the sensors and the controllers can be dramatically reduced while guaranteeing a satisfactory closed-loop performance [37]. Since the early seminal works on event-triggered control [3436], several different event-triggered mechanisms and control schemes have been proposed, and quite a few theoretical results have appeared that investigate the event-triggered control systems; see, for example, $[38,39]$ and references cited therein. Over the past few decades, a wealth of constructive complementary contributions have been made toward this interesting topic; to mention a few, the approaches of eventtriggered model predictive control for discrete-time linear systems are presented in [40]; later in [41], the problem of event-triggered model predictive control for continuous-time nonlinear systems subject to bounded disturbances is dealt with. The contribution of [35] is that a novel event-triggered PID controller is designed, and the PID controller does not compute the control input until the change of the measurement signal is large enough. The work of [42] proposes some improvements of the event-triggered PID controller presented in [35], whereas it should be pointed out that most of the previous results are concerned with the state-feedback control schemes, which is on the presupposition that all states of the plant can be measured. However, in many control applications full state measurements are not always available for feedback; therefore, in such cases, it is of great significance to investigate the event-triggered output feedback control strategies, some results of which can be found in [42].

Moreover, it should be noted that, among the existing literatures on event-triggered control, most results are focused on linear systems, while the problem of eventtriggered control for switched delay systems with exogenous disturbance has not been yet addressed, which motivates the current study. In this paper, the problem of observer-based event-triggered control for continuous-time switched linear systems with time-varying delay and norm-bounded disturbance is investigated, and we opt for a continuous-time eventtrigger to "observe" the "event," defined as some error signals exceeding a given threshold, to determine the updating of the controller. Besides, we utilize the state observer to generate the state estimates; then the observer-based event-triggered controller is designed to guarantee the finite-time boundedness and finite-time stabilization of the resulting closed-loop system. The main contributions of this paper lie in the following. (i) The event-triggered control scheme is firstly applied to switched systems subject to time-varying delay and normbounded exogenous disturbance. (ii) Sufficient conditions for finite-time boundedness and finite-time stabilization of switched delay systems are given. Besides, the analysis on minimum interevent interval is performed, and the lower bound of the minimum interevent interval is obtained. (iii) Combining event-triggering signal and subsystem switching signal together, a time interval partition algorithm is presented.

The rest of this paper is organized as follows: Section 2 contains the problem statement and preliminaries; Section 3 presents finite-time boundedness, finite-time stabilization, and minimum interevent interval performance for switched delay systems; Section 4 provides a numerical example to verify the effectiveness of the proposed results; concluding remarks are given in Section 5.

Notations. The following notations are used throughout the paper. $\mathbb{N}$ represents the set of natural numbers, $\mathbb{N}^{+}$stands for positive integer, $\mathbb{R}^{n}$ denotes the $n$ dimensional Euclidean space, and $\mathbb{R}^{m \times n}$ is the set of all $m \times n$ matrices. For any real number $x,[x]$ denotes the integer part of $x . X>Y$ $(X \geq Y, X<Y, X \leq Y)$, where $X$ and $Y$ are both symmetric matrices, meaning that $X-Y$ is positive (positive-semi, negative, negative-semi) definite. The identity matrix of order $n$ is denoted as $I_{n}$ (or, simply, $I$ if no confusion arises). The superscript " $T$ " is used to stand for matrix transposition. For a symmetric block matrix, we use $\star$ to denote the terms introduced by symmetry. For any symmetric matrix $P, \lambda_{\max }(P)$ and $\lambda_{\text {min }}(P)$ denote the maximum and minimum eigenvalues of matrix $P$, respectively. $\|v\|$ is the Euclidean norm of vector $v,\|v\|=\left(v^{T} v\right)^{1 / 2}$, while $\|A\|$ is spectral norm of matrix $A$, $\|A\|=\left[\lambda_{\max }\left(A^{T} A\right)\right]^{1 / 2}$. Matrices, if their dimensions are not explicitly stated, are assumed to have compatible dimensions for algebraic operations.

\section{Problem Statement and Preliminaries}

As shown in Figure 1, the event-triggered control system considered in this paper can be divided into the following three modules: (i) the physical plant and observer; (ii) the event-trigger; (iii) the event-triggered controller. 


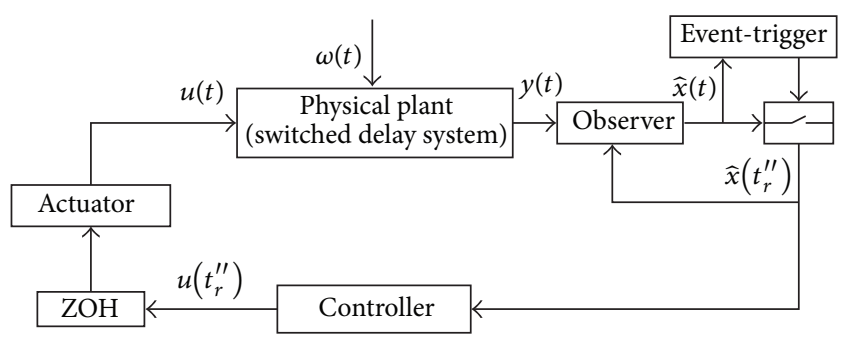

FIGURE 1: Event-triggered control system.

2.1. Physical Plant and Observer. Consider a continuoustime switched linear system with time-varying delay and exogenous disturbance described by

$$
\begin{aligned}
\dot{x}(t)= & A_{\sigma(t)} x(t)+A_{d \sigma(t)} x(t-\tau(t))+B_{\sigma(t)} u(t) \\
& +G_{\sigma(t)} \omega(t), \\
y(t)= & C_{\sigma(t)} x(t), \\
x(t)= & \phi(t),
\end{aligned}
$$

$$
t \in[-\tau, 0], \tau>0
$$

where $x \in \mathbb{R}^{n}$ is the system state vector, $u \in \mathbb{R}^{m}$ represents the control input vector, and $y \in \mathbb{R}^{q}$ denotes the output vector. $\omega(t) \in \mathbb{R}^{p}$ is the time-varying exogenous disturbance input satisfying $\int_{0}^{\infty} \omega^{T}(t) \omega(t) d t \leq d, d \geq 0 . \tau(t)$ denotes the time-varying delay satisfying $0 \leq \tau(t)<\tau, \dot{\tau}(t) \leq$ $h<1 . \phi(t)$ is a continuous vector-valued initial function on $[-\tau, 0]$ which specifies the initial values of states of system (1). $A_{\sigma(t)}, A_{d \sigma(t)}, B_{\sigma(t)}, C_{\sigma(t)}, G_{\sigma(t)}$ are all well-known constant system matrices with appropriate dimensions, and the pairs $\left(A_{\sigma(t)}, B_{\sigma(t)}\right)$ and $\left(C_{\sigma(t)}, A_{\sigma(t)}\right)$ are controllable and observable, respectively. $\sigma(t):[0, \infty) \rightarrow \mathcal{N}=\{1,2, \ldots, N\}$ is the switching signal which is a piecewise constant function and specifies which subsystem functions at a certain time instant. Corresponding to the switching signal $\sigma(t)$, we have the following switching sequence:

$$
\begin{aligned}
\zeta= & \left\{x\left(t_{0}^{\prime}\right) ;\left(i_{0}, t_{0}^{\prime}\right),\left(i_{1}, t_{1}^{\prime}\right), \ldots,\left(i_{k}, t_{k}^{\prime}\right), \ldots \mid i_{k}\right. \\
& \in \mathcal{N}, k \in \mathbb{N}\},
\end{aligned}
$$

where $t_{0}^{\prime}, x\left(t_{0}^{\prime}\right)$ represent the initial time instant and state, respectively, and $t_{0}^{\prime}<t_{1}^{\prime}<\cdots<t_{k}^{\prime}<\cdots$. When $t \in\left[t_{k}^{\prime}, t_{k+1}^{\prime}\right)$, the $i_{k}$ th subsystem is activated.

For the switched linear system (1), the purpose of this paper is to design the Luenberger observer, which can generate the accurate state estimates of system (1) by using the measured output $y(t)$. Then, an event-triggered controller will be synthesized based on the estimates to stabilize the resulting closed-loop system.
The Luenberger observer for system (1) is introduced as follows:

$$
\begin{aligned}
\dot{\hat{x}}(t)= & A_{\sigma(t)} \hat{x}(t)+A_{d \sigma(t)} \hat{x}(t-\tau(t))+B_{\sigma(t)} u(t) \\
& +L_{\sigma(t)}(y(t)-\widehat{y}(t)), \\
\hat{y}(t)= & C_{\sigma(t)} \hat{x}(t),
\end{aligned}
$$

where $\hat{x}(t) \in \mathbb{R}^{n}$ is the observer state and $L_{\sigma(t)}$ is the observer gain to be appropriately designed.

Assumption 1. The state trajectories of system (1) do not jump at switching instants; that is, the trajectories of $x(t)$ are everywhere continuous.

2.2. Event-Trigger and Event-Triggered Controller. The eventtrigger detects the event-triggered condition to determine whether an "event" is generated or not. Once an event happens, the event-trigger will transmit the latest state estimates to the event-triggered controller. Specifically, we have the following event-trigger instant sequence:

$$
\vartheta=\left\{\left[t_{0}^{\prime \prime}, t_{1}^{\prime \prime}\right),\left[t_{1}^{\prime \prime}, t_{2}^{\prime \prime}\right), \ldots,\left[t_{r}^{\prime \prime}, t_{r+1}^{\prime \prime}\right), \ldots \mid r \in \mathbb{N}\right\}
$$

with $t_{r}^{\prime \prime}<t_{r+1}^{\prime \prime}$. Without loss of generality, we assume that the first event happens at time instant $t_{0}^{\prime \prime}$ and $t_{0}^{\prime \prime}=0$. With the estimates $\hat{x}(t)$ of system states $x(t)$ sampled at time instant $t_{r}^{\prime \prime}$, the next sampling instant $t_{r+1}^{\prime \prime}$ can be determined by the event-trigger, and in this paper, we focus on the continuoustime event-trigger which is given by

$$
t_{r+1}^{\prime \prime}=\inf \left\{t>t_{r}^{\prime \prime}:\|e(t)\| \geq \gamma(t)\right\}, \quad \forall r \in \mathbb{N},
$$

where $e(t)=\widehat{x}(t)-\widehat{x}\left(t_{0}^{\prime \prime}\right)$ and $\gamma(t)=\sqrt{\epsilon^{-\beta t}+\epsilon_{0}}$ is the exponentially decreasing event threshold with given parameters $\epsilon>1,0 \leq \beta<1$, and $\epsilon_{0} \geq 0$.

In this paper, the controller is event-triggered, and the control inputs are determined on the basis of the sampled observer states, which can be given by

$$
u(t)=K_{\sigma(t)} \widehat{x}\left(t_{r}^{\prime \prime}\right), \quad t \in\left[t_{r}^{\prime \prime}, t_{r+1}^{\prime \prime}\right), \forall r \in \mathbb{N},
$$

where $K_{\sigma(t)}$ is the feedback gain with appropriate dimension. Note that, in the event-triggered control scenario, at sampling time instant $t_{r}^{\prime \prime}$, the controller in (6) will receive the sampled estimated states $\widehat{x}\left(t_{r}^{\prime \prime}\right)$, which will be held constant until next event is generated at time instant $t_{r+1}^{\prime \prime}$. Thereby, the control input in (6) is only updated at the sampling instants. Hence, a zero-order holder $(\mathrm{ZOH})$ is equipped in the system in order to keep the control signal continuous.

Before proceeding with the main results, we will first recall the following definitions and lemmas, which play an important role in the proof of the main results.

Definition 2 (see [43]). Given time instants $t$ and $T$ such that $0 \leq t \leq T$, let $N_{\sigma}(t, T)$ denote the switching number of $\sigma(t)$ over $(t, T)$, if

$$
N_{\sigma}(t, T) \leq N_{0}+\frac{(T-t)}{\tau_{a}}
$$


holds for $\tau_{a}>0$ and an integer $N_{0} \geq 0$; then $\tau_{a}$ is called an average dwell time.

Definition 3 (see [44]). Given four positive constants $d, T_{f}, c_{1}$, and $c_{2}$ such that $c_{1}<c_{2}$, a matrix $R>0$, and a switching signal $\sigma(t)$. If

$$
\begin{gathered}
\sup _{-\tau \leq \theta \leq 0} x^{T}(\theta) R x(\theta)<c_{1} \Longrightarrow \\
x^{T}(t) R x(t)<c_{2},
\end{gathered}
$$

$$
\forall t \in\left[0, T_{f}\right]
$$

holds, then system (1) with $u(t) \equiv 0$ is said to be finitetime bounded with respect to $\left(c_{1}, c_{2}, T_{f}, d, \tau, R, \sigma\right)$. If (8) holds for any switching signal $\sigma(t)$, system (1) with $u(t) \equiv 0$ is said to be uniformly finite-time bounded with respect to $\left(c_{1}, c_{2}, T_{f}, d, \tau, R\right)$.

Definition 4 (see [44]). Given four positive constants $d, T_{f}$, $c_{1}$, and $c_{2}$, such that $c_{1}<c_{2}$, a matrix $R>0$, and a switching signal $\sigma(t)$. If

$$
\begin{gathered}
\sup _{-\tau \leq \theta \leq 0} x^{T}(\theta) R x(\theta)<c_{1} \Longrightarrow \\
x^{T}(t) R x(t)<c_{2},
\end{gathered}
$$

$$
\forall t \in\left[0, T_{f}\right]
$$

holds, then system (1) with observer-based control (6) is said to be finite-time stabilizable with respect to $\left(c_{1}, c_{2}, T_{f}\right.$, $d, \tau, R, \sigma)$.

Lemma 5 (see [45]). Let $W \in \mathbb{R}^{n \times n}$ be a symmetric matrix, and let $x \in \mathbb{R}^{n}$; then the following inequality holds:

$$
\lambda_{\min }(W) x^{T} x \leq x^{T} W x \leq \lambda_{\max }(W) x^{T} x
$$

Lemma 6 ((Schur complement), see [46]). Given symmetric matrix $S=\left[\begin{array}{ll}S_{11} & S_{12} \\ S_{21} & S_{22}\end{array}\right]$, where $S_{11} \in R^{r \times r}$, then the following three conditions are equivalent: (1) $S<0$; (2) $S_{11}<0, S_{22}-$ $S_{12}^{T} S_{11}^{-1} S_{12}<0$; (3) $S_{22}<0, S_{11}-S_{12} S_{22}^{-1} S_{12}^{T}<0$.

Lemma 7 (see [47]). Assume $A \in \mathbb{R}^{n \times n}$ is Hurwitz; then there exists a positive scalar $c>0$ such that

$$
\left\|e^{A t}\right\| \leq c e^{\left(\lambda_{\max }(A) / 2\right) t},
$$

where $\lambda_{\max }(A)=\max _{i}\left\{\operatorname{Re}\left(\lambda_{i}(A)\right)\right\}$.

\section{Main Results}

3.1. Modeling of Closed-Loop System. Define the estimation error $\tilde{x}(t)=x(t)-\widehat{x}(t)$. For the time period $t \in\left[t_{r}^{\prime \prime}, t_{r+1}^{\prime \prime}\right)$, the plant and error systems can be rewritten as follows:

$$
\begin{aligned}
\dot{x}(t)= & A_{\sigma(t)} x(t)+A_{d \sigma(t)} x(t-\tau(t)) \\
& +B_{\sigma(t)} K_{\sigma(t)} \hat{x}\left(t_{r}^{\prime \prime}\right)+G_{\sigma(t)} \omega(t) \\
= & A_{\sigma(t)} x(t)+A_{d \sigma(t)} x(t-\tau(t)) \\
& +B_{\sigma(t)} K_{\sigma(t)} \hat{x}\left(t_{r}^{\prime \prime}\right)+G_{\sigma(t)} \omega(t) \\
& +B_{\sigma(t)} K_{\sigma(t)}(x(t)-\tilde{x}(t)-\widehat{x}(t)) \\
= & \left(A_{\sigma(t)}+B_{\sigma(t)} K_{\sigma(t)}\right) x(t)+A_{d \sigma(t)} x(t-\tau(t)) \\
& -B_{\sigma(t)} K_{\sigma(t)} \tilde{x}(t)-B_{\sigma(t)} K_{\sigma(t)} e(t)+G_{\sigma(t)} \omega(t),
\end{aligned}
$$

where $e(t)=\hat{x}(t)-\widehat{x}\left(t_{r}^{\prime \prime}\right)$, and

$$
\begin{aligned}
\dot{\tilde{x}}(t)= & \left(A_{\sigma(t)}-L_{\sigma(t)} C_{\sigma(t)}\right) \tilde{x}(t)+A_{d \sigma(t)} \tilde{x}(t-\tau(t)) \\
& +G_{\sigma(t)} \omega(t) .
\end{aligned}
$$

Define $\xi(t)=\left[x^{T}(t), \widetilde{x}^{T}(t)\right]^{T}$; then we have the following augmented closed-loop system:

$$
\begin{aligned}
\dot{\xi}(t)= & \bar{A}_{\sigma(t)} \xi(t)+\bar{B}_{\sigma(t)} e(t)+\bar{A}_{d \sigma(t)} \xi(t-\tau(t)) \\
& +\bar{G}_{\sigma(t)} \omega(t),
\end{aligned}
$$

where

$$
\begin{aligned}
& \bar{A}_{\sigma(t)}=\left[\begin{array}{cc}
A_{\sigma(t)}+B_{\sigma(t)} K_{\sigma(t)} & -B_{\sigma(t)} K_{\sigma(t)} \\
0 & A_{\sigma(t)}-L_{\sigma(t)} C_{\sigma(t)}
\end{array}\right], \\
& \bar{B}_{\sigma(t)}=\left[\begin{array}{c}
-B_{\sigma(t)} K_{\sigma(t)} \\
0
\end{array}\right], \\
& \bar{A}_{d \sigma(t)}=\left[\begin{array}{cc}
A_{d \sigma(t)} & 0 \\
0 & A_{d \sigma(t)}
\end{array}\right], \\
& \bar{G}_{\sigma(t)}=\left[\begin{array}{l}
G_{\sigma(t)} \\
G_{\sigma(t)}
\end{array}\right] .
\end{aligned}
$$

3.2. Time Interval Partition. From (2) and (4), we know that there exist two different "switching signals" which partition the time axis into different consecutive tiny intervals, which makes the problem of event-triggered control for switched systems more interesting and challenging. To address this difficulty, it is desirable to partition the time axis afresh to obtain the "switching instants" denoted by $\left\{t_{n}\right\}_{n=0}^{\infty}$. As depicted in Figure 2, when $t \in\left[t_{k}^{\prime}, t_{k+1}^{\prime}\right)$, that is, the $\sigma\left(t_{k}^{\prime}\right)$ th subsystem is activated, and it is assumed that $t_{r}^{\prime \prime}$ is the latest event-trigger instant before $t_{k+1}^{\prime}$; obviously, there are two different cases of the location of $t_{r}^{\prime \prime}$, as illustrated in Figures 2 (a) and 2(b), respectively. Figure 2(a) shows that $t_{r}^{\prime \prime}>t_{k}^{\prime}$; 


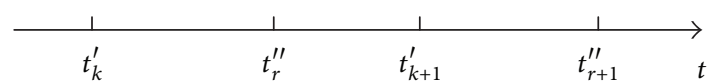

(a)

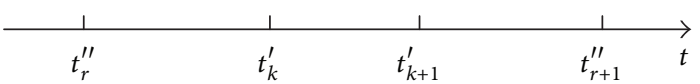

(b)

FIGURE 2: For $t \in\left[t_{k}^{\prime}, t_{k+1}^{\prime}\right)$, that is, the $\sigma\left(t_{k}^{\prime}\right)$ th subsystem is activated, let $t_{n+1}=t_{k+1}^{\prime} \cdot t_{n}$ is determined according to the relation between $t_{k}^{\prime}$ and the latest event-trigger instant before $t_{k+1}^{\prime}$ denoted by $t_{k}^{\prime \prime}$. (a) $t_{n}=\max \left\{t_{k}^{\prime \prime}, t_{k}^{\prime}\right\}=t_{k}^{\prime \prime}$; (b) $t_{n}=\max \left\{t_{k}^{\prime \prime}, t_{k}^{\prime}\right\}=t_{k}^{\prime}$.
(1) Given: $t_{n+1}=t_{k+1}^{\prime}, t_{0}=0$;
(2) for $i=n:-1: 1$, and $\forall p, q \in \mathbb{N}$, such that $t_{p}^{\prime}, t_{q}^{\prime \prime} \leq t_{i+1}$
(3) $t_{i}=t_{i+1}-\min \left\{t_{i+1}-t_{p}^{\prime}, t_{i+1}-t_{q}^{\prime \prime}\right\}$
(4) end for
(5) end

Algorithm 1: Computing "switching instants" $t_{n}$.

consequently, for the interval $\left[t_{r}^{\prime \prime}, t_{k+1}^{\prime}\right)$, the control input $u(t) \equiv u\left(t_{r}^{\prime \prime}\right)$; hence, for this interval, we can utilize the welldeveloped analysis approach of event-triggered control for linear systems. Then we can set $t_{n}=t_{r}^{\prime \prime}$ and $t_{n+1}=t_{k+1}^{\prime}$. Accordingly, we have $t_{n}=t_{k}^{\prime}$ and $t_{n+1}=t_{k+1}^{\prime}$ for Figure 2(b). The procedure of determining $t_{n}$ is given in Algorithm 1 .

Remark 8. On the basis of the above discuss, we can conclude that the set of the "switching instants" $\left\{t_{n} \mid n \in \mathbb{N}\right\}=\left\{t_{k}^{\prime} \mid k \in\right.$ $\mathbb{N}\} \cup\left\{t_{r}^{\prime \prime} \mid r \in \mathbb{N}\right\}$, if the subsystem switching instants $\left\{t_{k}^{\prime} \mid\right.$ $k \in \mathbb{N}\}$ and event-trigger instants $\left\{t_{r}^{\prime \prime} \mid r \in \mathbb{N}\right\}$ do not occur simultaneously. In the sequel, we denote by $\varrho(t)$ the value of "switching instants" $\left\{t_{n} \mid n \in \mathbb{N}\right\}$ at time instant $t$.

3.3. Finite-Time Boundedness Analysis. The following theorem indicates that the finite-time boundedness of the closedloop system (14) can be guaranteed by using the abovementioned event-triggered control scheme.

Theorem 9. For any $\sigma(t)=i \in \mathcal{N}=\{1,2, \ldots, N\}$, suppose that there exist matrices $P_{i}>0, Q_{i}>0$, let $\widetilde{P}_{i}=$ $R^{1 / 2} P_{i} R^{1 / 2}, \widetilde{Q}_{i}=R^{1 / 2} Q_{i} R^{1 / 2}$, and there exist constants $\alpha>$ $0, \mu \geq 1, \eta>0$ such that

$$
\begin{aligned}
& \Xi=\left[\begin{array}{cccc}
\bar{A}_{i}^{T} \widetilde{P}_{i}+\widetilde{P}_{i} \bar{A}_{i}+\widetilde{Q}_{i}-\alpha \widetilde{P}_{i} & \widetilde{P}_{i} \bar{A}_{d i} & \widetilde{P}_{i} \bar{B}_{i} & \widetilde{P}_{i} \bar{G}_{i} \\
\star & -(1-h) \widetilde{Q}_{i} & 0 & 0 \\
\star & \star & -I & 0 \\
\star & \star & \star & -\eta I
\end{array}\right] \\
& <0, \\
& e^{\alpha T_{f}}\left(\kappa_{2}+\tau \kappa_{3}\right) c_{1}+\rho_{1}+\rho_{2}+\rho_{3}<c_{2} \kappa_{1} .
\end{aligned}
$$

If the average dwell time of the "switching signal" $\varrho(t)$ satisfies

$$
\begin{aligned}
\tau_{a}>\tau_{a}^{*} \\
=\frac{T_{f} \ln \mu}{\ln \left(\kappa_{1} c_{2}-\rho_{1}-\rho_{2}-\rho_{3}\right)-\ln \left(c_{1} \kappa_{2}+\tau c_{1} \kappa_{3}\right)-\alpha T_{f}},
\end{aligned}
$$

then system (14) is finite-time bounded with respect to $\left(c_{1}, c_{2}, T_{f}, d, \tau, R, \varrho\right)$, where $\kappa_{1}=\min _{i \in \mathcal{N}}\left(\lambda_{\min }\left(P_{i}\right)\right), \kappa_{2}=$ $\max _{i \in \mathcal{N}}\left(\lambda_{\max }\left(P_{i}\right)\right), \kappa_{3}=\max _{i \in \mathcal{N}}\left(\lambda_{\max }\left(Q_{i}\right)\right)$, and

$$
\begin{aligned}
\rho_{1} & =\sum_{i=0}^{\left[T_{f} / \tau_{a}\right]} \mu^{i} e^{\alpha T_{f}}\left(\frac{e^{(-\alpha-\beta \ln \epsilon) t_{\left[T_{f} / \tau_{a}\right]-i}}}{\alpha+\beta \ln \epsilon}\right), \\
& \left.+\frac{\left(\epsilon_{0}+\eta d\right) e^{-\alpha t_{\left[T_{f} / \tau_{a}\right]-i}}}{\alpha}\right) \\
\rho_{2}= & -(\alpha+\beta \ln \epsilon)^{-1} \\
& \cdot \sum_{j=1}^{\left[T_{f} / \tau_{a}\right]}\left(\mu^{j} e^{-\beta \ln \epsilon t_{\left[T_{f} / \tau_{a}\right]-j+1}+\alpha\left(t_{\left[T_{f} / \tau_{a}\right]}-t_{\left[T_{f} / \tau_{a}\right]-j+1}\right)}\right. \\
& \left.-\frac{\epsilon_{0}+\eta d}{\alpha} \mu^{j} e^{\alpha\left(T_{f}-t_{\left[T_{f} / \tau_{a}\right]-j+1}\right)}\right), \\
\rho_{3}= & -(\alpha+\beta \ln \epsilon)^{-1} e^{-\beta \ln \epsilon T_{f}}-\frac{\epsilon_{0}+\eta d}{\alpha} .
\end{aligned}
$$

Proof. Choose the following Lyapunov-like function:

$$
V(t)=\xi^{T}(t) \widetilde{P}_{i} \xi(t)+\int_{t-\tau(t)}^{t} \xi^{T}(\theta) \widetilde{Q}_{i} \xi(\theta) d \theta .
$$

Taking the derivative of $V(t)$ with respect to $t$ along the trajectory of system (14) reads

$$
\begin{aligned}
\dot{V}(t) & =\dot{\xi}^{T}(t) \widetilde{P}_{i} \xi(t)+\xi^{T}(t) \widetilde{P}_{i} \dot{\xi}(t)+\xi^{T}(t) \widetilde{Q}_{i} \xi(t)-\xi^{T}(t-\tau(t)) \widetilde{Q}_{i} \xi(t-\tau(t))(1-\dot{\tau}(t)) \\
& =\xi^{T}(t)\left(\bar{A}_{i}^{T} \widetilde{P}_{i}+\widetilde{P}_{i} \bar{A}_{i}+\widetilde{Q}_{i}\right) \xi(t)+2 \xi^{T}(t-\tau(t)) \bar{A}_{d i}^{T} \widetilde{P}_{i} \xi(t)+2 e^{T}(t) \bar{B}_{i}^{T} \widetilde{P}_{i} \xi(t)+2 \omega^{T}(t) \bar{G}_{i}^{T} \widetilde{P}_{i} \xi(t)
\end{aligned}
$$




$$
\begin{aligned}
& -\xi^{T}(t-\tau(t)) \widetilde{Q}_{i} \xi(t-\tau(t))(1-\dot{\tau}(t)) \\
\leq & \xi^{T}(t)\left(\bar{A}_{i}^{T} \widetilde{P}_{i}+\widetilde{P}_{i} \bar{A}_{i}+\widetilde{Q}_{i}\right) \xi(t)+2 \xi^{T}(t-\tau(t)) \bar{A}_{d i}^{T} \widetilde{P}_{i} \xi(t)+2 e^{T}(t) \bar{B}_{i}^{T} \widetilde{P}_{i} \xi(t)+2 \omega^{T}(t) \bar{G}_{i}^{T} \widetilde{P}_{i} \xi(t) \\
& -\xi^{T}(t-\tau(t)) \widetilde{Q}_{i} \xi(t-\tau(t))(1-h) \\
= & {\left[\begin{array}{c}
\xi(t) \\
\xi(t-\tau(t)) \\
e(t) \\
\omega(t)
\end{array}\right]\left[\begin{array}{rrrr}
\bar{A}_{i}^{T} \widetilde{P}_{i}+\widetilde{P}_{i} \bar{A}_{i}+\widetilde{Q}_{i} & \widetilde{P}_{i} \bar{A}_{d i} & \widetilde{P}_{i} \bar{B}_{i} & \widetilde{P}_{i} \bar{G}_{i} \\
\star & -(1-h) \widetilde{Q}_{i} & 0 & 0 \\
\star & \star & 0 & 0 \\
\star & \star & \star & 0
\end{array}\right]\left[\begin{array}{c}
\xi(t) \\
\xi(t-\tau(t)) \\
e(t) \\
\omega(t)
\end{array}\right] . }
\end{aligned}
$$

According to (16), we have

$$
\begin{aligned}
\dot{V}(t) \leq & {\left[\begin{array}{c}
\xi(t) \\
\xi(t-\tau(t)) \\
e(t) \\
\omega(t)
\end{array}\right]^{T} \Xi\left[\begin{array}{c}
\xi(t) \\
\xi(t-\tau(t)) \\
e(t) \\
\omega(t)
\end{array}\right] } \\
+ & {\left.\left[\begin{array}{c}
\xi(t) \\
\xi(t-\tau(t)) \\
e(t) \\
\omega(t)
\end{array}\right]\right]^{T} } \\
& \cdot\left[\begin{array}{cccc}
\alpha \widetilde{P}_{i} & 0 & 0 & 0 \\
\star & 0 & 0 & 0 \\
\star & \star & I & 0 \\
\star & \star & \star & \eta I
\end{array}\right]\left[\begin{array}{c}
\xi(t) \\
\xi(t-\tau(t)) \\
e(t) \\
\omega(t)
\end{array}\right],
\end{aligned}
$$

which is equivalent to

$$
\begin{gathered}
\left.\dot{V}(t)-\left[\begin{array}{c}
\xi(t) \\
\xi(t-\tau(t)) \\
e(t) \\
\omega(t)
\end{array}\right]\right]^{T} \\
\cdot\left[\begin{array}{cccc}
\alpha \widetilde{P}_{i} & 0 & 0 & 0 \\
\star & 0 & 0 & 0 \\
\star & \star & I & 0 \\
\star & \star & \star & \eta I
\end{array}\right]\left[\begin{array}{c}
\xi(t) \\
\xi(t-\tau(t)) \\
e(t) \\
\omega(t)
\end{array}\right]<0, \\
\left.\leq\left[\begin{array}{c}
\xi(t) \\
\xi(t-\tau(t)) \\
e(t) \\
\omega(t)
\end{array}\right] \begin{array}{c}
\xi(t) \\
\xi(t-\tau(t)) \\
e(t) \\
\omega(t)
\end{array}\right]<0
\end{gathered}
$$

to continue

$$
\begin{aligned}
\dot{V}(t) & \leq \alpha \xi^{T}(t) \widetilde{P}_{i} \xi(t)+e^{T}(t) e(t)+\eta \omega^{T}(t) \omega(t) \\
& \leq \alpha V(t)+\|e(t)\|^{2}+\eta \omega^{T}(t) \omega(t) \\
& \leq \alpha V(t)+\gamma^{2}(t)+\eta \omega^{T}(t) \omega(t) .
\end{aligned}
$$

Integrating (25) from $t_{n}$ to $t$ gives

(23)

$V(t)$

$$
\begin{aligned}
\leq & V\left(t_{n}\right) e^{\int_{t_{n}}^{t} \alpha d s} \\
& +e^{\int_{t_{n}}^{t} \alpha d s} \int_{t_{n}}^{t}\left(\gamma^{2}(s)+\eta \omega^{T}(s) \omega(s)\right) e^{-\int_{t_{n}}^{s} \alpha d t} d s \\
= & V\left(t_{n}\right) e^{\alpha\left(t-t_{n}\right)}+\int_{t_{n}}^{t} e^{\alpha(t-s)} \gamma^{2}(s) d s \\
& +\int_{t_{n}}^{t} e^{\alpha(t-s)} \eta \omega^{T}(s) \omega(s) d s \\
\leq & V\left(t_{n}\right) e^{\alpha\left(t-t_{n}\right)}+e^{\alpha t} \int_{t_{n}}^{t} e^{(-\alpha-\beta \ln \epsilon) s} d s \\
& +\left(\epsilon_{0}+\eta d\right) e^{\alpha t} \int_{t_{n}}^{t} e^{-\alpha s} d s \\
= & V\left(t_{n}\right) e^{\alpha\left(t-t_{n}\right)} \\
& +e^{\alpha t}\left(\frac{e^{(-\alpha-\beta \ln \epsilon) t_{n}}}{\alpha+\beta \ln \epsilon}+\frac{\left(\epsilon_{0}+\eta d\right) e^{-\alpha t_{n}}}{\alpha}\right) \\
& -\frac{e^{-\beta \ln \epsilon t}}{\alpha+\beta \ln \epsilon}-\frac{\epsilon_{0}+\eta d}{\alpha} .
\end{aligned}
$$

Suppose at the instant $t_{n}, \sigma\left(t_{n}\right)=i, \sigma\left(t_{n}^{-}\right)=j, i, j \in \mathcal{N}$. Therefore, if the condition (17) holds, then

$$
V_{\sigma\left(t_{n}\right)}\left(t_{n}\right) \leq \mu V_{\sigma\left(t_{n}^{-}\right)}\left(t_{n}^{-}\right) .
$$


Hence, we can further obtain

$$
\begin{aligned}
& V(t) \leq V\left(t_{n}\right) e^{\alpha\left(t-t_{n}\right)}+e^{\alpha t}\left(\frac{e^{(-\alpha-\beta \ln \epsilon) t_{n}}}{\alpha+\beta \ln \epsilon}\right. \\
& \left.+\frac{\left(\epsilon_{0}+\eta d\right) e^{-\alpha t_{n}}}{\alpha}\right)-\frac{e^{-\beta \ln \epsilon t}}{\alpha+\beta \ln \epsilon}-\frac{\epsilon_{0}+\eta d}{\alpha} \\
& \leq \mu V\left(t_{n}^{-}\right) e^{\alpha\left(t-t_{n}\right)}+e^{\alpha t}\left(\frac{e^{(-\alpha-\beta \ln \epsilon) t_{n}}}{\alpha+\beta \ln \epsilon}\right. \\
& \left.+\frac{\left(\epsilon_{0}+\eta d\right) e^{-\alpha t_{n}}}{\alpha}\right)-\frac{e^{-\beta \ln \epsilon t}}{\alpha+\beta \ln \epsilon}-\frac{\epsilon_{0}+\eta d}{\alpha} \\
& \leq \mu\left(V\left(t_{n-1}\right) e^{\alpha\left(t_{n}^{-}-t_{n-1}\right)}+e^{\alpha t_{n}^{-}}\left(\frac{e^{(-\alpha-\beta \ln \epsilon) t_{n-1}}}{\alpha+\beta \ln \epsilon}\right.\right. \\
& \left.+\frac{\left(\epsilon_{0}+\eta d\right) e^{-\alpha t_{n-1}}}{\alpha}\right)-(\alpha+\beta \ln \epsilon)^{-1} e^{-\beta \ln \epsilon t_{n}^{-}} \\
& \left.-\frac{\epsilon_{0}+\eta d}{\alpha}\right) e^{\alpha\left(t-t_{n}\right)}+\left(\frac{e^{(-\alpha-\beta \ln \epsilon) t_{n}}}{\alpha+\beta \ln \epsilon}\right. \\
& \left.+\frac{\left(\epsilon_{0}+\eta d\right) e^{-\alpha t_{n}}}{\alpha}\right) e^{\alpha t}-(\alpha+\beta \ln \epsilon)^{-1} e^{-\beta \ln \epsilon t} \\
& -\frac{\epsilon_{0}+\eta d}{\alpha}=\mu V\left(t_{n-1}\right) e^{\alpha\left(t-t_{n-1}\right)} \\
& +\mu e^{\alpha t}\left(\frac{e^{(-\alpha-\beta \ln \epsilon) t_{n-1}}}{\alpha+\beta \ln \epsilon}+\frac{\left(\epsilon_{0}+\eta d\right) e^{-\alpha t_{n-1}}}{\alpha}\right)-\mu(\alpha \\
& +\beta \ln \epsilon)^{-1} e^{-\beta \ln \epsilon t_{n}^{-}+\alpha\left(t-t_{n}\right)}-\mu \frac{\epsilon_{0}+\eta d}{\alpha} e^{\alpha\left(t-t_{n}\right)} \\
& +\left(\frac{e^{(-\alpha-\beta \ln \epsilon) t_{n}}}{\alpha+\beta \ln \epsilon}+\frac{\left(\epsilon_{0}+\eta d\right) e^{-\alpha t_{n}}}{\alpha}\right) e^{\alpha t}-(\alpha+\beta \\
& \cdot \ln \epsilon)^{-1} e^{-\beta \ln \epsilon t}-\frac{\epsilon_{0}+\eta d}{\alpha} \leq \mu^{2}\left(V\left(t_{n-2}\right)\right. \\
& \cdot e^{\alpha\left(t_{n-1}^{-}-t_{n-2}\right)}+e^{\alpha t_{n-1}^{-}}\left(\frac{e^{(-\alpha-\beta \ln \epsilon) t_{n-2}}}{\alpha+\beta \ln \epsilon}\right. \\
& \left.+\frac{\left(\epsilon_{0}+\eta d\right) e^{-\alpha t_{n-2}}}{\alpha}\right)-(\alpha+\beta \ln \epsilon)^{-1} e^{-\beta \ln \epsilon t_{n-1}^{-}} \\
& \left.-\frac{\epsilon_{0}+\eta d}{\alpha}\right) e^{\alpha\left(t-t_{n-1}\right)}+\mu e^{\alpha t}\left(\frac{e^{(-\alpha-\beta \ln \epsilon) t_{n-1}}}{\alpha+\beta \ln \epsilon}\right. \\
& \left.+\frac{\left(\epsilon_{0}+\eta d\right) e^{-\alpha t_{n-1}}}{\alpha}\right)-\mu(\alpha+\beta \ln \epsilon)^{-1} \\
& \cdot e^{-\beta \ln \epsilon t_{n}^{-}+\alpha\left(t-t_{n}\right)}-\mu \frac{\epsilon_{0}+\eta d}{\alpha} e^{\alpha\left(t-t_{n}\right)}+\left(\frac{e^{(-\alpha-\beta \ln \epsilon) t_{n}}}{\alpha+\beta \ln \epsilon}\right. \\
& \left.+\frac{\left(\epsilon_{0}+\eta d\right) e^{-\alpha t_{n}}}{\alpha}\right) e^{\alpha t}-(\alpha+\beta \ln \epsilon)^{-1} e^{-\beta \ln \epsilon t}
\end{aligned}
$$

$$
\begin{aligned}
& -\frac{\epsilon_{0}+\eta d}{\alpha}=\mu^{2} V\left(t_{n-2}\right) e^{\alpha\left(t-t_{n-2}\right)} \\
& +\mu^{2} e^{\alpha t}\left(\frac{e^{(-\alpha-\beta \ln \epsilon) t_{n-2}}}{\alpha+\beta \ln \epsilon}+\frac{\left(\epsilon_{0}+\eta d\right) e^{-\alpha t_{n-2}}}{\alpha}\right) \\
& -\mu^{2}(\alpha+\beta \ln \epsilon)^{-1} e^{-\beta \ln \epsilon t_{n-1}^{-}+\alpha\left(t-t_{n-1}\right)}-\mu^{2} \frac{\epsilon_{0}+\eta d}{\alpha} \\
& \cdot e^{\alpha\left(t-t_{n-1}\right)}+\mu e^{\alpha t}\left(\frac{e^{(-\alpha-\beta \ln \epsilon) t_{n-1}}}{\alpha+\beta \ln \epsilon}\right. \\
& \left.+\frac{\left(\epsilon_{0}+\eta d\right) e^{-\alpha t_{n-1}}}{\alpha}\right)-\mu(\alpha+\beta \ln \epsilon)^{-1} \\
& \cdot e^{-\beta \ln \epsilon t_{n}^{-}+\alpha\left(t-t_{n}\right)}-\mu \frac{\epsilon_{0}+\eta d}{\alpha} e^{\alpha\left(t-t_{n}\right)}+\left(\frac{e^{(-\alpha-\beta \ln \epsilon) t_{n}}}{\alpha+\beta \ln \epsilon}\right. \\
& \left.+\frac{\left(\epsilon_{0}+\eta d\right) e^{-\alpha t_{n}}}{\alpha}\right) e^{\alpha t}-(\alpha+\beta \ln \epsilon)^{-1} e^{-\beta \ln \epsilon t} \\
& -\frac{\epsilon_{0}+\eta d}{\alpha} \cdots \leq \mu^{n} V\left(t_{0}\right) e^{\alpha t} \\
& +\sum_{i=0}^{n} \mu^{i} e^{\alpha t}\left(\frac{e^{(-\alpha-\beta \ln \epsilon) t_{n-i}}}{\alpha+\beta \ln \epsilon}+\frac{\left(\epsilon_{0}+\eta d\right) e^{-\alpha t_{n-i}}}{\alpha}\right)-(\alpha \\
& +\beta \ln \epsilon)^{-1} \sum_{j=1}^{n}\left(\mu^{j} e^{-\beta \ln \epsilon t_{n-j+1}^{-}+\alpha\left(t-t_{n-j+1}\right)}\right. \\
& \left.-\frac{\epsilon_{0}+\eta d}{\alpha} \mu^{j} e^{\alpha\left(t-t_{n-j+1}\right)}\right)-(\alpha+\beta \ln \epsilon)^{-1} e^{-\beta \ln \epsilon t} \\
& -\frac{\epsilon_{0}+\eta d}{\alpha} \leq \mu^{S} V\left(t_{0}\right) e^{\alpha T_{f}} \\
& +\sum_{i=0}^{n} \mu^{i} e^{\alpha T_{f}}\left(\frac{e^{(-\alpha-\beta \ln \epsilon) t_{n-i}}}{\alpha+\beta \ln \epsilon}+\frac{\left(\epsilon_{0}+\eta d\right) e^{-\alpha t_{n-i}}}{\alpha}\right) \\
& -(\alpha+\beta \ln \epsilon)^{-1} \sum_{j=1}^{n}\left(\mu^{j} e^{-\beta \ln \epsilon t_{n-j+1}^{-}+\alpha\left(t_{n}-t_{n-j+1}\right)}\right. \\
& \left.-\frac{\epsilon_{0}+\eta d}{\alpha} \mu^{j} e^{\alpha\left(T_{f}-t_{n-j+1}\right)}\right)-(\alpha+\beta \ln \epsilon)^{-1} e^{-\beta \ln \epsilon T_{f}} \\
& -\frac{\epsilon_{0}+\eta d}{\alpha}
\end{aligned}
$$

where $S$ denotes the "switching" number of $\varrho(t)$ over $\left(0, T_{f}\right)$, which means $S \leq T_{f} / \tau_{a}$; therefore, it can be obtained that

$$
V(t) \leq e^{\alpha T_{f}} \mu^{T_{f} / \tau_{a}} V\left(t_{0}\right)+\rho_{1}+\rho_{2}+\rho_{3} .
$$

On the other hand,

$$
\begin{aligned}
V(t) & \geq \xi^{T}(t) \widetilde{P}_{i} \xi^{T}(t) \geq \lambda_{\min }\left(P_{i}\right) \xi^{T}(t) R \xi(t) \\
& \geq \kappa_{1} \xi^{T}(t) R \xi(t)
\end{aligned}
$$




$$
\begin{aligned}
& V(0)=\xi^{T}(0) \widetilde{P}_{\sigma(0)} \xi(0)+\int_{-\tau(0)}^{0} \xi^{T}(\theta) \widetilde{Q}_{\sigma(0)} \xi(\theta) d \theta \\
& \quad \leq\left(\lambda_{\max }\left(P_{i}\right)+\tau \lambda_{\max }\left(Q_{i}\right)\right) \sup _{-\tau \leq \theta \leq 0}\left\{\xi^{T}(\theta) R \xi(\theta)\right\} \\
& \quad \leq\left(\kappa_{2}+\tau \kappa_{3}\right) c_{1} .
\end{aligned}
$$

Combining (29), (30), and (31), it can be deduced that

$$
\begin{aligned}
\xi^{T}(t) R \xi(t) & \leq \frac{e^{\alpha T_{f}} \mu^{T_{f} / \tau_{a}} V\left(t_{0}\right)+\rho_{1}+\rho_{2}+\rho_{3}}{\kappa_{1}} \\
& \leq \frac{e^{\alpha T_{f}} \mu^{T_{f} / \tau_{a}}\left(\kappa_{2}+\tau \kappa_{3}\right) c_{1}+\rho_{1}+\rho_{2}+\rho_{3}}{\kappa_{1}} .
\end{aligned}
$$

If $\mu=1$, then according to (18), we have

$$
\xi^{T}(t) R \xi(t) \leq \frac{e^{\alpha T_{f}}\left(\kappa_{2}+\tau \kappa_{3}\right) c_{1}+\rho_{1}+\rho_{2}+\rho_{3}}{\kappa_{1}}<c_{2}
$$

If $\mu>1$, according to (19), one can get

$$
\begin{aligned}
& \frac{T_{f}}{\tau_{a}} \\
& <\frac{\ln \left(\kappa_{1} c_{2}-\rho_{1}-\rho_{2}-\rho_{3}\right)-\ln \left(c_{1} \kappa_{2}+c_{1} \tau \kappa_{3}\right)-\alpha T_{f}}{\ln \mu} .
\end{aligned}
$$

Substituting (34) to (32) yields

$$
\begin{aligned}
& \xi^{T}(t) R \xi(t) \\
& <\frac{e^{\alpha T_{f}\left(\kappa_{1} c_{2}-\rho_{1}-\rho_{2}-\rho_{3}\right) /\left(c_{1} \kappa_{2}+c_{1} \tau \kappa_{3}\right)} e^{-\alpha T_{f}}\left(\kappa_{2}+\tau \kappa_{3}\right) c_{1}+\rho_{1}+\rho_{2}+\rho_{3}}{\kappa_{1}} \\
& =c_{2} .
\end{aligned}
$$

Then according to Definition 3, we can conclude that the finite-time boundedness of the resulting closed-loop system (14) can be guaranteed; thus, this proof is completed.

Remark 10. In Theorem 9, the meaning of average dwell time is somewhat different from the commonly used, due to the complexity of the problem considered here. Theorem 9 shows that, in order to guarantee the finite-time bounded quality of event-triggered switched control systems, the switching frequency of subsystems and event-trigger should have an upper bound over a finite-time interval.

Now, based on Theorem 9, the following corollary is obtained to give some conditions which guarantee uniform finite-time boundedness of system (14).
Corollary 11. For any $\sigma(t)=i \in \mathcal{N}=\{1,2, \ldots, N\}$, let $\widetilde{P}=$ $R^{1 / 2} P R^{1 / 2}, \widetilde{Q}=R^{1 / 2} Q R^{1 / 2}$, suppose there exist matrices $P>$ $0, Q>0$, and constants $\alpha \geq 1, \mu>1, \eta>0$ such that

$$
\begin{aligned}
\Psi & =\left[\begin{array}{cccc}
\bar{A}_{i}^{T} \widetilde{P}+\widetilde{P} \bar{A}_{i}+\widetilde{Q}-\alpha \widetilde{P} & \widetilde{P} \bar{A}_{d i} & \widetilde{P} \bar{B}_{i} & \widetilde{P} \bar{G}_{i} \\
\star & -(1-h) \widetilde{Q} & 0 & 0 \\
\star & \star & -I & 0 \\
\star & \star & \star & -\eta I
\end{array}\right] \\
& <0, \\
e^{\alpha T_{f}} & \left(\lambda_{\max }(P)+\tau \lambda_{\max }(Q)\right) c_{1}+\rho_{1}+\rho_{2}+\rho_{3}
\end{aligned}
$$

and then according to Definition 3, system (14) is uniformly finite-time bounded with respect to $\left(c_{1}, c_{2}, T_{f}, d, \tau, R\right)$.

Proof. Choose a Lyapunov-like function:

$$
V(t)=\xi^{T}(t) \widetilde{P} \xi(t)+\int_{t-\tau(t)}^{t} \xi^{T}(\theta) \widetilde{Q} \xi(\theta) d \theta .
$$

Substituting $\widetilde{P}_{i}, \widetilde{Q}_{i}$ with $\widetilde{P}, \widetilde{Q}$ into the proof procedure of Theorem 9 , it is easy to get the conclusion.

3.4. Finite-Time Stabilization Analysis. Next, let us consider the finite-time stabilization problem for system (14) based on the above results. The following theorem gives the conditions that can guarantee system (14) is finite-time stabilizable.

Theorem 12. Given positive constants $c_{1}, c_{2}, T_{f}, d, \tau$, a positive definite matrix $R$, system (14) is finite-time stabilizable with respect to $\left(c_{1}, c_{2}, T_{f}, d, \tau, R, \varrho\right)$, if the average dwell time of the "switching signal" $\varrho(t)$ satisfies (19), and the feedback gain $K_{i}$ is selected according to $B_{i} K_{i}=P_{1 i}^{-1} X_{i}$, the observer gain $L_{i}$ is selected according to $L_{i} C_{i}=P_{2 i}^{-1} Y_{i}$, and for any $\sigma(t)=i \in \mathcal{N}=$ $\{1,2, \ldots, N\}$, there exist matrices $P_{1 i}>0, P_{2 i}>0, Q_{1 i}>0$, $Q_{2 i}>0, X_{i}>0, Y_{i}>0$ and constant $\eta>0$ such that

$\Theta$

$$
=\left[\begin{array}{ccccccc}
\Theta_{11} & -X_{i} & P_{1 i} A_{d i} & 0 & -X_{i} & P_{1 i} G_{i} & I \\
\star & \Theta_{22} & 0 & P_{2 i} A_{d i} & 0 & P_{2 i} G_{i} & 0 \\
\star & \star & -(1-h) Q_{1 i} & 0 & 0 & 0 & 0 \\
\star & \star & \star & -(1-h) Q_{2 i} & 0 & 0 & 0 \\
\star & \star & \star & \star & -I & 0 & 0 \\
\star & \star & \star & \star & \star & \eta I & 0 \\
\star & \star & \star & \star & \star & \star & -Q_{1 i}^{-1}
\end{array}\right]
$$

$<0$,

where

$$
\begin{aligned}
& \Theta_{11}=A_{i}^{T} P_{1 i}+X_{i}^{T}+P_{1 i} A_{i}+X_{i}-\alpha P_{1 i} \\
& \Theta_{22}=A_{i}^{T} P_{2 i}-Y_{i}^{T}+P_{2 i} A_{i}-Y_{i}+Q_{2 i}-\alpha P_{2 i}
\end{aligned}
$$


Proof. If condition (38) holds, then substituting $B_{i} K_{i}=$ $P_{1 i}^{-1} X_{i}, L_{i} C_{i}=P_{2 i}^{-1} Y_{i}$ into (16) and in view of Lemma 6, we can get

$$
\left[\begin{array}{cccc}
\bar{A}_{i}^{T} \widetilde{P}_{i}+\widetilde{P}_{i} \bar{A}_{i}+\widetilde{Q}_{i}-\alpha \widetilde{P}_{i} & \widetilde{P}_{i} \bar{A}_{d i} & \widetilde{P}_{i} \bar{B}_{i} & \widetilde{P}_{i} \bar{G}_{i} \\
\star & -(1-h) \widetilde{Q}_{i} & 0 & 0 \\
\star & \star & -I & 0 \\
\star & \star & \star & -\eta I
\end{array}\right]
$$

$<0$,

where

$$
\begin{aligned}
\widetilde{P}_{i} & =\left[\begin{array}{cc}
P_{1 i} & 0 \\
0 & P_{2 i}
\end{array}\right], \\
\widetilde{Q}_{i} & =\left[\begin{array}{cc}
Q_{1 i} & 0 \\
0 & Q_{2 i}
\end{array}\right] .
\end{aligned}
$$

The rest proof procedure is similar to that of Theorem 9, and due to limited space, we will not cover that again; thus, this proof is completed.

Remark 13. In the proofs of Theorems 9 and 12, it is not required that $\dot{V}(t)<0$, which is required for asymptotical stability of switched systems via a common Lyapunov function. However, conditions (18) and (19), which are essential for finite-time stabilization of switched systems, are not needed for asymptotical stability. Moreover, the switched system concerned in this paper is a certain dynamical system, whereas some valuable results for uncertain dynamical systems are presented in chapter 9 of [48], where the problem of absolute parameter stability for uncertain singular perturbation systems is thoroughly investigated and several different sorts of Lyapunov functions are constructed.

3.5. The Minimum Interevent Interval. To exclude the Zeno behavior of sampling, it is needed to show there always exists a nonzero lower bound of the minimum interevent interval. The following theorem shows that there exists a positive lower bound of the minimum interevent interval.

Theorem 14. With the sampling instants determined by (5), the minimum interevent interval is lower bounded by a positive scalar.

Proof. For any $r \in \mathbb{N}$, let $t_{r}^{\prime \prime}$ be a sampling instant. Then, in the time interval $\left[t_{r}^{\prime \prime}, t_{r+1}^{\prime \prime}\right), \widehat{x}\left(t_{r}^{\prime \prime}\right)$ is constant. In light of the definition of $e(t)$ in (12), for $t \in\left[t_{r}^{\prime \prime}, t_{r+1}^{\prime \prime}\right)$, we have

$$
\begin{aligned}
\dot{e}(t)= & A_{i} \hat{x}(t)+A_{d i} \hat{x}(t-\tau(t))+B_{i} u(t) \\
& +L_{i}(y(t)-\hat{y}(t)) \\
= & A_{i} e(t)+A_{d i} \hat{x}(t-\tau(t))+\left(A_{i}+B_{i} K_{i}\right) \hat{x}\left(t_{r}^{\prime \prime}\right) \\
& +L_{i} C_{i} \tilde{x}(t),
\end{aligned}
$$

which yields that

$$
\begin{aligned}
& e(t)=e^{\int_{t_{r}^{\prime \prime}}^{t} A_{\sigma(s)} d s} e\left(t_{r}^{\prime \prime}\right) \\
& +\int_{t_{r}^{\prime \prime}}^{t} e^{\int_{t_{r}^{\prime \prime}}^{t} A_{\sigma(s)} d s+\int_{s}^{t_{r}^{\prime \prime}} A_{\sigma(t)} d t}\left[A_{d \sigma(s)} \widehat{x}(s-\tau(s))\right. \\
& +\left(A_{\sigma(s)}+B_{\sigma(s)} K_{\sigma(s)}\right) \hat{x}\left(t_{r}^{\prime \prime}\right) \\
& \left.+L_{\sigma(s)} C_{\sigma(s)} \tilde{x}(s)\right] d s \\
& =\int_{t_{r}^{\prime \prime}}^{t} e^{\int_{t_{r}^{\prime \prime}}^{t} A_{\sigma(s)} d s+\int_{s}^{t_{r}^{\prime \prime}} A_{\sigma(t)} d t}\left[A_{d \sigma(s)} \widehat{x}(s-\tau(s))\right. \\
& +\left(A_{\sigma(s)}+B_{\sigma(s)} K_{\sigma(s)}\right) \hat{x}\left(t_{r}^{\prime \prime}\right) \\
& \left.+L_{\sigma(s)} C_{\sigma(s)} \widetilde{x}(s)\right] d s .
\end{aligned}
$$

Moreover, noticing (13), we have

$$
\begin{aligned}
\dot{\tilde{x}}(t)= & \left(A_{\sigma(t)}-L_{\sigma(t)} C_{\sigma(t)}\right) \tilde{x}(t)+A_{d \sigma(t)} \tilde{x}(t-\tau(t)) \\
& +G_{\sigma(t)} \omega(t),
\end{aligned}
$$

which leads to

$$
\begin{aligned}
& \widetilde{x}(t)=e^{\int_{0}^{t}\left(A_{\sigma(s)}-L_{\sigma(s)} C_{\sigma(s)}\right) d s} \widetilde{x}(0) \\
& +e^{\int_{0}^{t}\left(A_{\sigma(s)}-L_{\sigma(s)} C_{\sigma(s)}\right) d s} \int_{0}^{t}\left[A_{d \sigma(s)} \widetilde{x}(s-\tau(s))\right. \\
& \left.+G_{\sigma(s)} \omega(s)\right] e^{-\int_{0}^{s}\left(A_{\sigma(t)}-L_{\sigma(t)} C_{\sigma(t)}\right) d t} d s,
\end{aligned}
$$

where $\tilde{x}(0)$ is the initial estimate error, and it is natural to assume that $\|\widetilde{x}(0)\|$ is bounded.

Then, we have

$$
\begin{aligned}
& \|e(t)\|=\| \int_{t_{r}^{\prime \prime}}^{t} e^{\int_{t_{r}^{\prime \prime}}^{t} A_{\sigma(s)} d s+\int_{s}^{t_{r}^{\prime \prime}} A_{\sigma(t)} d t}\left(A_{d \sigma(s)} \hat{x}(s-\tau(s))\right. \\
& +\left(A_{\sigma(s)}+B_{\sigma(s)} K_{\sigma(s)}\right) \hat{x}\left(t_{k}\right) \\
& \left.+L_{\sigma(s)} C_{\sigma(s)} \widetilde{x}(s)\right) d s \| \\
& \leq \int_{t_{r}^{\prime \prime}}^{t} e^{\left\|\int_{t_{r}^{\prime \prime}}^{t} A_{\sigma(s)} d s+\int_{s}^{t_{t}^{\prime \prime}} A_{\sigma(t)} d t\right\|} \| A_{d \sigma(s)} \widehat{x}(s-\tau(s)) \\
& +\left(A_{\sigma(s)}+B_{\sigma(s)} K_{\sigma(s)}\right) \hat{x}\left(t_{r}^{\prime \prime}\right) \\
& +L_{\sigma(s)} C_{\sigma(s)} \tilde{x}(s) \| d s \\
& \leq \int_{t_{r}^{\prime \prime}}^{t} e^{\|A\|(t-s)}\left(\left\|A_{d \sigma(s)} \widehat{x}(s-\tau(s))\right\|\right. \\
& +\left\|\left(A_{\sigma(s)}+B_{\sigma(s)} K_{\sigma(s)}\right) \hat{x}\left(t_{r}^{\prime \prime}\right)\right\| \\
& \left.+\left\|L_{\sigma(s)} C_{\sigma(s)} \tilde{x}(s)\right\|\right) d s \leq \int_{t_{r}^{\prime \prime}}^{t} e^{\|A\|(t-s)}\left(\left\|A_{d}\right\|\right. \\
& \cdot\left\|\lambda_{1}\left(t, t_{r}^{\prime \prime}\right)\right\|+\|A+B K\|\left\|\widehat{x}\left(t_{r}^{\prime \prime}\right)\right\|+\|L C\|
\end{aligned}
$$




$$
\begin{aligned}
& \cdot\|\tilde{x}(s)\|) d s \leq\left(\left\|A_{d}\right\|\left\|\lambda_{1}\left(t, t_{r}^{\prime \prime}\right)\right\|+\| A\right. \\
& \left.+B K\|\| \widehat{x}\left(t_{r}^{\prime \prime}\right) \|\right) \int_{t_{r}^{\prime \prime}}^{t} e^{\|A\|(t-s)} d s+\|L C\| \int_{t_{r}^{\prime \prime}}^{t} e^{\|A\|(t-s)} \\
& \cdot\left(c e^{\left(\max _{i \in \mathcal{N}}\left\{\lambda_{\max }\left(A_{i}-L_{i} C_{i}\right)\right\} / 2\right) t}\|\widetilde{x}(0)\|\right. \\
& +c e^{\left(\max _{i \in \mathcal{N}}\left\{\lambda_{\max }\left(A_{i}-L_{i} C_{i}\right)\right\} / 2\right) t}\left(\left\|A_{d}\right\|\left\|\lambda_{2}\left(t, t_{r}^{\prime \prime}\right)\right\|\right. \\
& \left.+\sqrt{d}\|G\|) \int_{0}^{s} e^{\|A-L C\| t} d t\right) d s \leq\left(\left\|A_{d}\right\|\right. \\
& \left.\cdot\left\|\lambda_{1}\left(t, t_{r}^{\prime \prime}\right)\right\|+\|A+B K\|\left\|\widehat{x}\left(t_{r}^{\prime \prime}\right)\right\|\right) \int_{t_{r}^{\prime \prime}}^{t} e^{\|A\|(t-s)} d s \\
& +\|L C\| c e^{\left(\max _{i \in \mathcal{N}}\left\{\lambda_{\max }\left(A_{i}-L_{i} C_{i}\right)\right\} / 2\right) t}\|\widetilde{x}(0)\| \\
& \cdot \int_{t_{r}^{\prime \prime}}^{t} e^{\|A\|(t-s)} d s+\|L C\| \\
& \cdot c e^{\left(\max _{i \in \mathcal{N}}\left\{\lambda_{\max }\left(A_{i}-L_{i} C_{i}\right)\right\} / 2\right) t}\left(\left\|A_{d}\right\|\left\|\lambda_{2}\left(t, t_{r}^{\prime \prime}\right)\right\|\right. \\
& +\sqrt{d}\|G\|) \frac{e^{\|A-L C\| t}}{\|A-L C\|} \int_{t_{r}^{\prime \prime}}^{t} e^{\|A\|(t-s)} d s=\varphi\left(t, t_{r}^{\prime \prime}\right) \\
& \cdot \int_{t_{r}^{\prime \prime}}^{t} e^{\|A\|(t-s)} d s,
\end{aligned}
$$

where

$$
\begin{aligned}
& \varphi\left(t, t_{r}^{\prime \prime}\right)=\left\|A_{d}\right\|\left\|\lambda_{1}\left(t, t_{r}^{\prime \prime}\right)\right\|+\|A+B K\|\left\|\widehat{x}\left(t_{r}^{\prime \prime}\right)\right\| \\
& +\|L C\| c e^{\left(\max _{i \in \mathscr{N}}\left\{\lambda_{\max }\left(A_{i}-L_{i} C_{i}\right)\right\} / 2\right) t}\|\widetilde{x}(0)\|+\|L C\| \\
& \cdot c e^{\left(\max _{i \in \mathcal{N}}\left\{\lambda_{\max }\left(A_{i}-L_{i} C_{i}\right)\right\} / 2\right) t}\left(\left\|A_{d}\right\|\left\|\lambda_{2}\left(t, t_{r}^{\prime \prime}\right)\right\|\right. \\
& +\sqrt{d}\|G\|) \frac{e^{\|A-L C\| t}}{\|A-L C\|},
\end{aligned}
$$

with

$$
\begin{aligned}
\|A\| & =\max _{i \in \mathcal{N}}\left\{\left\|A_{i}\right\|\right\}, \\
\left\|A_{d}\right\| & =\max _{i \in \mathcal{N}}\left\{\left\|A_{d i}\right\|\right\}, \\
\|G\| & =\max _{i \in \mathcal{N}}\left\{\left\|G_{i}\right\|\right\}, \\
\|A+B K\| & =\max _{i \in \mathcal{N}}\left\{\left\|A_{i}+B_{i} K_{i}\right\|\right\}, \\
\|L C\| & =\max _{i \in \mathcal{N}}\left\{\left\|L_{i} C_{i}\right\|\right\}, \\
\|A-L C\| & =\max _{i \in \mathcal{N}}\left\{\left\|A_{i}-L_{i} C_{i}\right\|\right\}, \\
\left\|\lambda_{1}\left(t, t_{r}^{\prime \prime}\right)\right\| & =\max _{t_{r}^{\prime \prime}-\tau \leq s \leq t}\{\|\widehat{x}(s)\|\}, \\
\left\|\lambda_{2}\left(t, t_{r}^{\prime \prime}\right)\right\| & =\max _{t_{r}^{\prime \prime}-\tau \leq s \leq t}\{\tilde{x}(s)\} .
\end{aligned}
$$

By doing so, and assuming $\|A\| \neq 0$, we have

$$
\|e(t)\| \leq \frac{\varphi\left(t, t_{r}^{\prime \prime}\right)}{\|A\|}\left(e^{\|A\|\left(t-t_{r}^{\prime \prime}\right)}-1\right) .
$$

According to the definition of sampling instants (5), the next event will not be generated before $\|e(t)\|=\gamma(t)$. Therefore, a lower bound on the interevent interval denoted by $\Delta t=t-t_{r}^{\prime \prime}$ can be determined by

$$
\frac{\varphi\left(t, t_{r}^{\prime \prime}\right)}{\|A\|}\left(e^{\|A\| \Delta t}-1\right)=\sqrt{\epsilon^{-\beta\left(\Delta t+t_{r}^{\prime \prime}\right)}+\epsilon_{0}},
$$

which means that, for any given sampling instant $t_{r}^{\prime \prime}, \Delta t$ cannot be zero; thus, $\Delta t>0$, which completes this proof.

Remark 15. In Theorem 14, the existence of the positive lower bound of the minimum interevent interval $\Delta t_{\min }$ can be guaranteed. Moreover, from (50), it can be seen that the following inequality holds:

$$
\frac{\varphi\left(t, t_{r}^{\prime \prime}\right)}{\|A\|}\left(e^{\|A\| \Delta t}-1\right) \geq \sqrt{\epsilon_{0}}
$$

One thus can derive

$$
\Delta t \geq \frac{1}{\|A\|} \ln \frac{\varphi\left(t, t_{r}^{\prime \prime}\right)+\sqrt{\epsilon_{0}}\|A\|}{\varphi\left(t, t_{r}^{\prime \prime}\right)} .
$$

\section{Numerical Examples}

In this section, we will present a numerical example to show the validness of our results. The following two-mode switched delay system is considered.

Mode 1

$$
\begin{aligned}
A_{1} & =\left[\begin{array}{ccc}
-1.5 & -1.2 & -2.0 \\
0.5 & -1.3 & 0.3 \\
-1.2 & 0.8 & -1.5
\end{array}\right], \\
A_{d 1} & =\left[\begin{array}{lll}
0.1 & 0.2 & 0.1 \\
0.1 & 0.2 & 0.3 \\
0.3 & 0.1 & 0.2
\end{array}\right], \\
B_{1} & =\left[\begin{array}{lll}
0.8 & 0.5 & 1.5
\end{array}\right]^{T}, \\
C_{1} & =\left[\begin{array}{lll}
1 & 2 & 1
\end{array}\right], \\
G_{1} & =\left[\begin{array}{lll}
0.2 & 0.5 & 0.3
\end{array}\right]^{T} .
\end{aligned}
$$


Mode 2

$$
\begin{aligned}
A_{2} & =\left[\begin{array}{ccc}
-1.8 & -1.5 & -1.2 \\
0.3 & -1.3 & 0.5 \\
-0.5 & 1.2 & -1.5
\end{array}\right], \\
A_{d 2} & =\left[\begin{array}{lll}
0.3 & 0.2 & 0.1 \\
0.1 & 0.2 & 0.1 \\
0.2 & 0.1 & 0.3
\end{array}\right], \\
B_{2} & =\left[\begin{array}{lll}
0.4 & 0.5 & 0.2
\end{array}\right]^{T}, \\
C_{2} & =\left[\begin{array}{lll}
2 & 1 & 1
\end{array}\right], \\
G_{2} & =\left[\begin{array}{lll}
0.2 & 0.3 & 0.4
\end{array}\right]^{T} .
\end{aligned}
$$

In addition, the corresponding parameters are given as follows:

$$
\begin{aligned}
T_{f} & =15, \\
R & =I, \\
c_{1} & =5, \\
c_{2} & =10, \\
\alpha & =1, \\
\beta & =0.8, \\
\epsilon & =15+e, \\
\epsilon_{0} & =0.005, \\
\mu & =1.5, \\
\eta & =0.5, \\
\tau & =0.8, \\
h & =0.02, \\
d & =0.01, \\
\omega(t) & =0.02 e^{-t} .
\end{aligned}
$$

The initial values of the system states and the observers are selected as $[0.5,0,-2]^{T},[1,0,-2]^{T}$, respectively. The system state and the observer responses are shown in Figures 3 and 4, respectively, and Figure 5 depicts the trajectory of $x^{T}(x) R x(t)$, which illustrates that the switched system is finite-time bounded with respect to $(5,10,15,0.01,0.8, I, \varrho)$. The switching signal is shown in Figure 6, and if the switching is too frequent, it is possible that the switched system is not finite-time bounded any more. The curves of $\|e(t)\|$ and $\gamma(t)$ are plotted in Figure 7, corresponding to which, Figure 8 depicts the interevent intervals.

Remark 16. It should be pointed out that, in Figure 7, at the trigger instants, the value of $\|e(t)\|$ may be a little larger than $\gamma(t)$, due to the discrete feature of computer simulation.

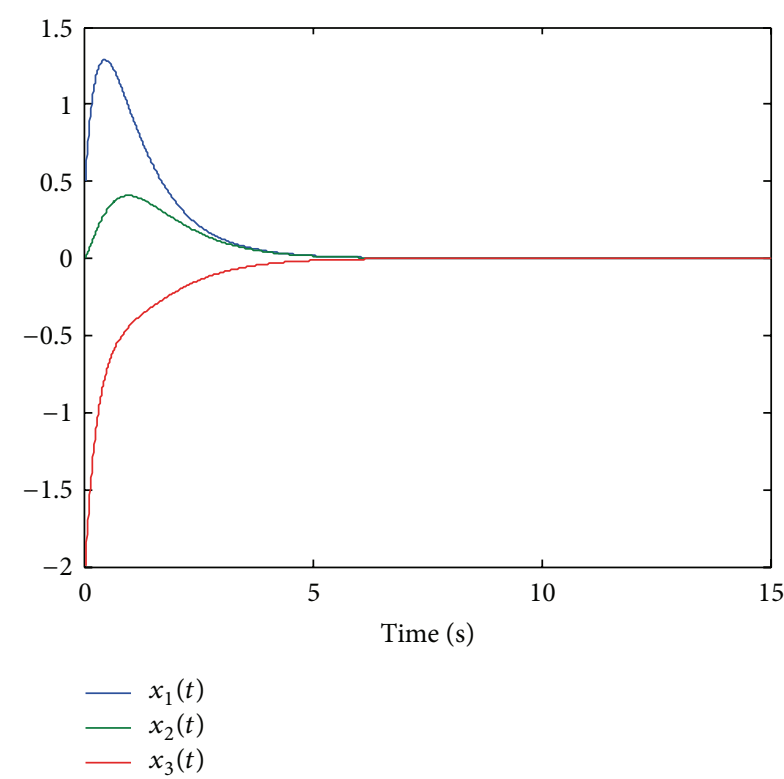

FIGURE 3: The state trajectory of the switched system.

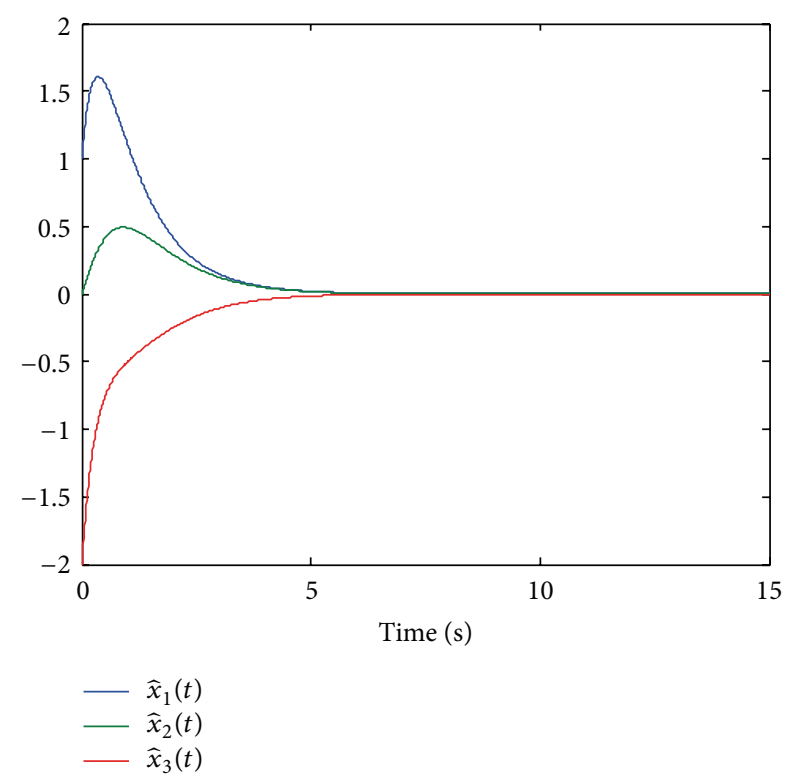

FIGURE 4: The estimated state trajectory of the switched system.

To obtain more precise simulation performance, a shorter simulation step is needed, which will inevitably make the simulation time become longer. Hence, it is desirable to achieve a compromise between simulation precision and simulation time, which is often encountered as carrying out a computer simulation.

\section{Conclusions}

In this paper, the design problem of the observed-based event-triggered control has been addressed for switched linear systems with time-varying delay and norm-bounded 


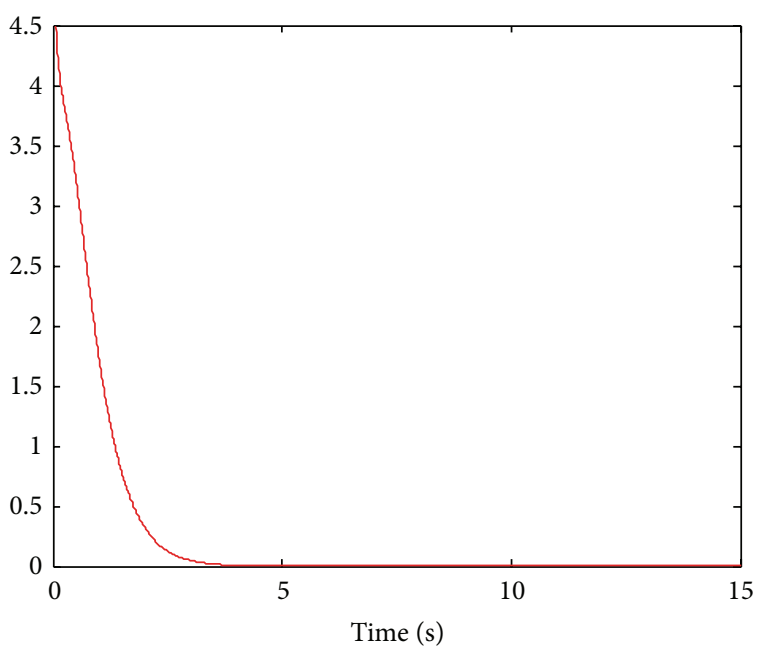

$-x^{T}(t) R x(t)$

Figure 5: The trajectory of $x^{T}(t) R x(t)$.

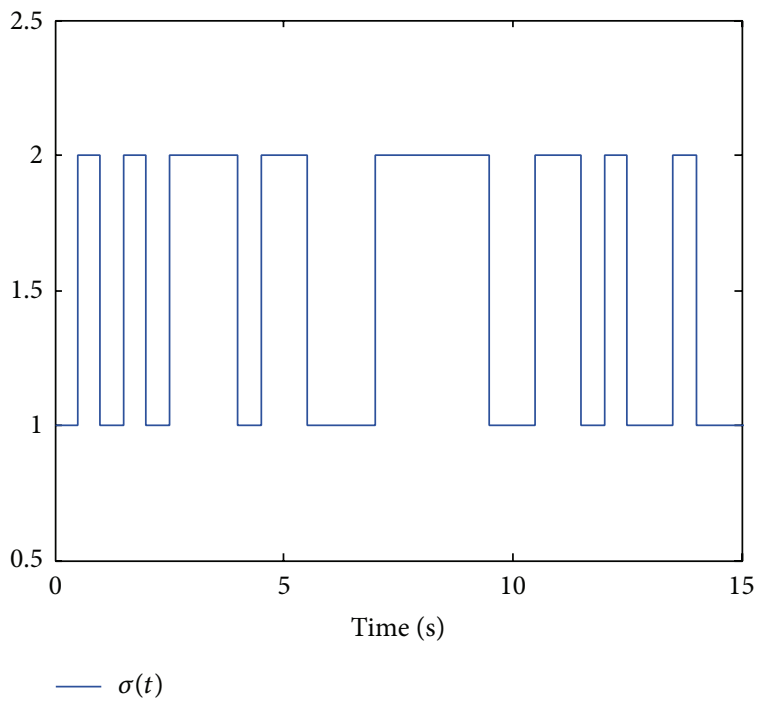

Figure 6: The switching signal $\sigma(t)$.

disturbance under continuous-time event-trigger. Unlike sampled-data control systems, the controller will not be updated until some error signal exceeds a well set threshold. It has also been demonstrated that the finite-time boundedness and finite-time stabilization of the closed-loop system can be guaranteed, and the lower bound of the minimum interevent interval has been proved to be positive to preclude the Zeno behavior of sampling. Finally, a numerical example has been given to verify the effectiveness of the achieved design approaches.

\section{Competing Interests}

The authors declare that they have no competing interests.

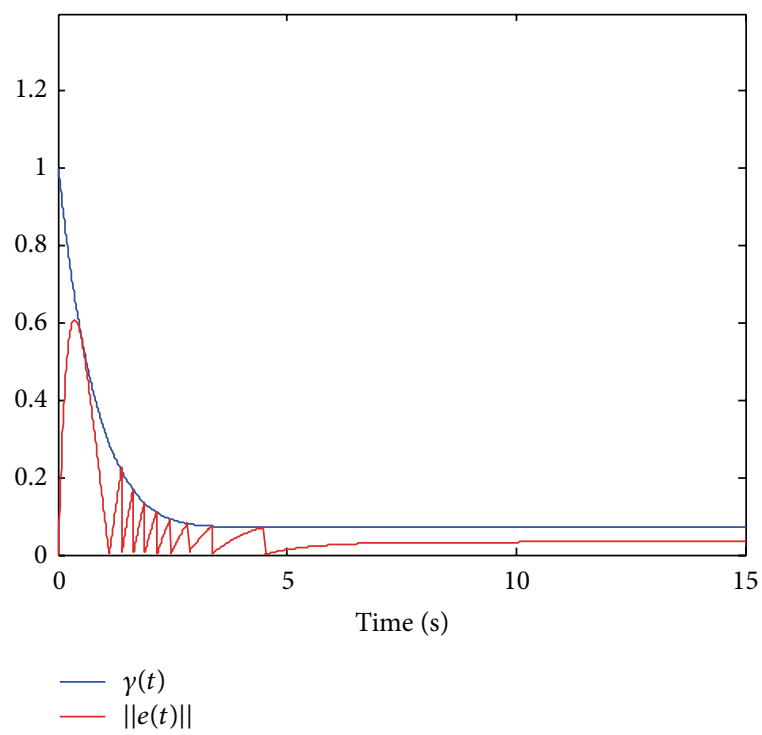

Figure 7: $\|e(t)\|$ and $\gamma(t)$.

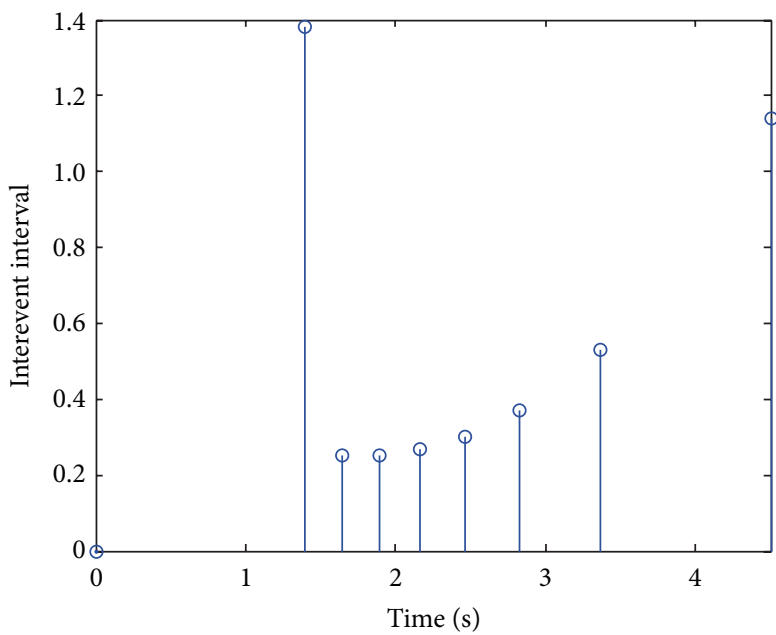

FiguRE 8: Interevent intervals.

\section{Acknowledgments}

This work is supported by the Fundamental Research Funds for the Central Universities under Grant WK2100100024.

\section{References}

[1] X. Zhao, L. Zhang, P. Shi, and H. R. Karimi, "Robust control of continuous-time systems with state-dependent uncertainties and its application to electronic circuits," IEEE Transactions on Industrial Electronics, vol. 61, no. 8, pp. 4161-4170, 2014.

[2] W. Zhang, L. Yu, and S. Yin, "A switched system approach to $H_{\infty}$ control of networked control systems with time-varying delays," Journal of the Franklin Institute, vol. 348, pp. 165-178, 2011.

[3] T. Kroger, On-Line Trajectory Generation in Robotic Systems, Springer, Berlin, Germany, 2010. 
[4] B. Lu, F. Wu, and S. Kim, "Switching LPV control of an F-16 aircraft via controller state reset," IEEE Transactions on Control Systems Technology, vol. 14, no. 2, pp. 267-277, 2006.

[5] L. Qin, G. Ma, Z. Chu, and G. Wu, "Modeling and control of greenhouse temperature-humidity system based on grey prediction model," Transactions of the Chinese Society of Agriculture Engineering, vol. 32, supplement 1, pp. 233-241, 2016.

[6] G. Chen and Y. Yang, "Finite-time stability of switched positive linear systems," International Journal of Robust and Nonlinear Control, vol. 24, no. 1, pp. 179-190, 2014.

[7] R. Rakkiyappan, A. Chandrasekar, S. Lakshmanan, and J. H. Park, "Exponential stability for markovian jumping stochastic BAM neural networks with mode-dependent probabilistic time-varying delays and impulse control," Complexity, vol. 20, no. 3, pp. 39-65, 2015.

[8] X. Liu, G. Ma, X. Jiang, and H. Xi, " $H_{\infty}$ stochastic synchronization for master-slave semi-Markovian switching system via sliding mode control," Complexity, 2015.

[9] Y. Chen and W. Zheng, "Stability analysis and control for switched stochastic delayed systems," International Journal of Robust and Nonlinear Control, vol. 26, no. 2, pp. 303-328, 2016.

[10] Y. Dong and F. Yang, "Finite-time stability and boundedness of switched nonlinear time-delay systems under state-dependent switching," Complexity, vol. 21, no. 2, pp. 267-275, 2015.

[11] X. Liu and H. Xi, "On exponential stability of neutral delay Markovian jump systems with nonlinear perturbations and partially unknown transition rates," International Journal of Control, Automation and Systems, vol. 12, no. 1, pp. 1-11, 2014

[12] X. Liu, X. Yu, and H. Xi, "Finite-time synchronization of neutral complex networks with Markovian switching based on pinning controller," Neurocomputing, vol. 153, pp. 148-158, 2015.

[13] Y. Yang, C. Xiang, and T. H. Lee, "Sufficient and necessary conditions for the stability of second-order switched linear systems under arbitrary switching," International Journal of Control, vol. 85, no. 12, pp. 1977-1995, 2012.

[14] G. Chen, Y. Yang, and Q. Pan, "Finite time stability analysis of switched systems with stable and unstable subsystems," Asian Journal of Control, vol. 16, no. 4, pp. 1224-1228, 2014.

[15] C. Liu, Q. Han, C. Li, C. Li, and Q. Zhang, "Reachability and controllability of linear switched impulsive systems," IET Control Theory and Applications, vol. 7, no. 9, pp. 1294-1299, 2013.

[16] L. Yu, G. Zheng, D. Boutat, and J.-P. Barbot, "Observability and observer design for a class of switched systems," IET Control Theory \& Applications, vol. 5, no. 9, pp. 1113-1119, 2011.

[17] D. Mincarelli, A. Pisano, T. Floquet, and E. Usai, "Uniformly convergent sliding mode-based observation for switched linear systems," International Journal of Robust and Nonlinear Control, 2015.

[18] X. Lin, H. Du, and S. Li, "Finite-time boundedness and $L_{2^{-}}$ gain analysis for switched delay systems with norm-bounded disturbance," Applied Mathematics and Computation, vol. 217, no. 12, pp. 5982-5993, 2011

[19] H. Liu, Y. Shen, and X. Zhao, "Delay-dependent observer-based $H_{\infty}$ finite-time control for switched systems with time-varying delay," Nonlinear Analysis: Hybrid Systems, vol. 6, no. 3, pp. 885898, 2012.

[20] P. Dorato, "Short time stability in linear time-varying systems," in Proceedings of the IRE International Convention Record Part 4, pp. 83-87, New York, NY, USA, 1961.
[21] Y. Wang, X. Shi, Z. Zuo, Y. Liu, and M. Z. Q. Chen, "Finite-time stability analysis of impulsive discrete-time switched systems with nonlinear perturbation," International Journal of Control, vol. 87, no. 11, pp. 2365-2371, 2014.

[22] W. Xiang and J. Xiao, "Finite-time stability and stabilisation for switched linear systems," International Journal of Systems Science, vol. 44, no. 2, pp. 384-400, 2013.

[23] Y. Wang, X. Shi, G. Wang, and Z. Zuo, "Finite-time stability for continuous-time switched systems in the presence of impulse effects," IET Control Theory and Applications, vol. 6, no. 11, pp. 1741-1744, 2012.

[24] W.-A. Zhang, L. Yu, and S. Yin, "A switched system approach to $H_{\infty}$ control of networked control systems with time-varying delays," Journal of the Franklin Institute, vol. 348, no. 2, pp. 165178, 2011.

[25] X.-M. Sun, G.-P. Liu, W. Wang, and D. Rees, "Stability analysis for networked control systems based on average dwell time method," International Journal of Robust and Nonlinear Control, vol. 20, no. 15, pp. 1774-1784, 2010.

[26] J.-D. Chen, C.-D. Yang, C.-H. Lien, and J.-H. Horng, "New delay-dependent non-fragile $H_{\infty}$ observer-based control for continuous time-delay systems," Information Sciences, vol. 178, no. 24, pp. 4699-4706, 2008.

[27] J. Buisson, P.-Y. Richard, and H. Cormerais, "On the stabilisation of switching electrical power converters," in Hybrid Systems: Computation and Control, M. Morari and L. Thiele, Eds., vol. 3414 of Lecture Notes in Computer Science, pp. 184197, Springer, Berlin, Germany, 2005.

[28] C. Meyer, S. Schröder, and R. W. De Doncker, "Solid-state circuit breakers and current limiters for medium-voltage systems having distributed power systems," IEEE Transactions on Power Electronics, vol. 19, no. 5, pp. 1333-1340, 2004.

[29] M. Mahmoud, "Delay-dependent $H_{\infty}$ filtering of a class of switched discrete-time state delay systems," Signal Processing, vol. 88, pp. 2709-2719, 2008.

[30] M. Mazo Jr., A. Anta, and P. Tabuada, "An ISS self-triggered implementation of linear controllers," Automatica, vol. 46, no. 8, pp. 1310-1314, 2010.

[31] W. P. M. H. Heemels and M. C. F. Donkers, "Model-based periodic event-triggered control for linear systems," Automatica, vol. 49, no. 3, pp. 698-711, 2013.

[32] X. Liu and H. Xi, "Exponential synchronization for neutral complex dynamical networks with interval mode-dependent delays and sampled data," Mathematical Problems in Engineering, vol. 2013, Article ID 639580, 21 pages, 2013.

[33] W. P. Heemels, M. C. Donkers, and A. Teel, "Periodic eventtriggered control for linear systems," IEEE Transactions on Automatic Control, vol. 58, no. 4, pp. 847-861, 2013.

[34] W. P. M. H. Heemels, R. J. A. Gorter, A. Van Zijl et al., "Asynchronous measurement and control: a case study on motor synchronization," Control Engineering Practice, vol. 7, no. 12, pp. 1467-1482, 1999.

[35] K. Arzén, “A simple event-based PID conroller," IFAC World Congress, vol. 18, pp. 423-428, 1999.

[36] K. Aström and B. Bernhardsson, "Comparison of periodic and event based sampling for first-order stochastic systems," in Proceedings of the IFAC World Congress, pp. 301-306, Beijing, China, July 1999.

[37] D. Lehmann and J. Lunze, "Extension and experimental evaluation of an event-based state-feedback approach," Control Engineering Practice, vol. 19, no. 2, pp. 101-112, 2011. 
[38] P. J. Gawthrop and L. Wang, "Event-driven intermittent control," International Journal of Control, vol. 82, no. 12, pp. 22352248, 2009.

[39] W. P. Heemels, J. H. Sandee, and P. P. J. van den Bosch, “Analysis of event-driven controllers for linear systems," International Journal of Control, vol. 81, no. 4, pp. 571-590, 2008.

[40] A. Eqtami, D. V. Dimarogonas, and K. J. Kyriakopoulos, "Eventtriggered control for discrete-time systems," in Proceedings of the American Control Conference (ACC '10), pp. 4719-4724, Baltimore, Md, USA, July 2010.

[41] H. Li and Y. Shi, "Event-triggered robust model predictive control of continuous-time nonlinear systems," Automatica, vol. 50, no. 5, pp. 1507-1513, 2014.

[42] D. Lehmann and J. Lunze, "Event-based output-feedback control," in Proceedings of the 19th Mediterranean Conference on Control and Automation (MED '11), pp. 982-987, Corfu, Greece, June 2011.

[43] J. Hespanha and A. Morse, "Stability of switched systems with average dwell-time," in Proceedings of the 38th IEEE Conference on Decision and Control, pp. 2655-2660, Phoenix, Ariz, USA, 1999.

[44] H. Liu, Y. Shen, and X. Zhao, "Delay-dependent observer-based $H_{\infty}$ finite-time control for switched systems with time-varying delay," Nonlinear Analysis: Hybrid Systems, vol. 6, no. 3, pp. 885898, 2012.

[45] X. Liu, X. Yu, G. Ma, and H. Xi, “On sliding mode control for networked control systems with semi-Markovian switching and random sensor delays," Information Sciences, vol. 337-338, pp. 44-58, 2016.

[46] S. Boyd, L. Ghaoui, E. Feron, and V. Balakrishnan, Linear Matrix Inequalities in System and Control Theorey, Society for Industrial and Applied Mathematics, Philadelphia, Pa, USA, 1994.

[47] W. Zhang, Stability analysis of network control systems [Ph.D. thesis], Department of Electrical Engineering and Computer Science, Case Western Reserve University, Cleveland, Ohio, USA, 2001.

[48] A. Martynyuk, Y. Martynyuk-Chernienko, and Z. Sun, Uncertain Dynamical Systems: Stability and Motion Control, Science Press, 2011. 


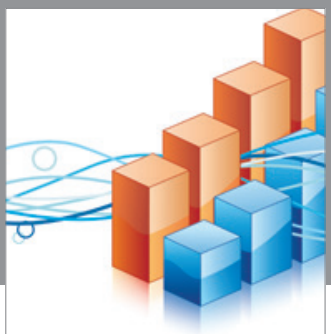

Advances in

Operations Research

vatem alat4

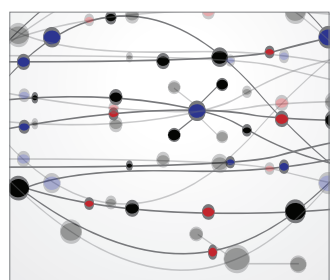

\section{The Scientific} World Journal
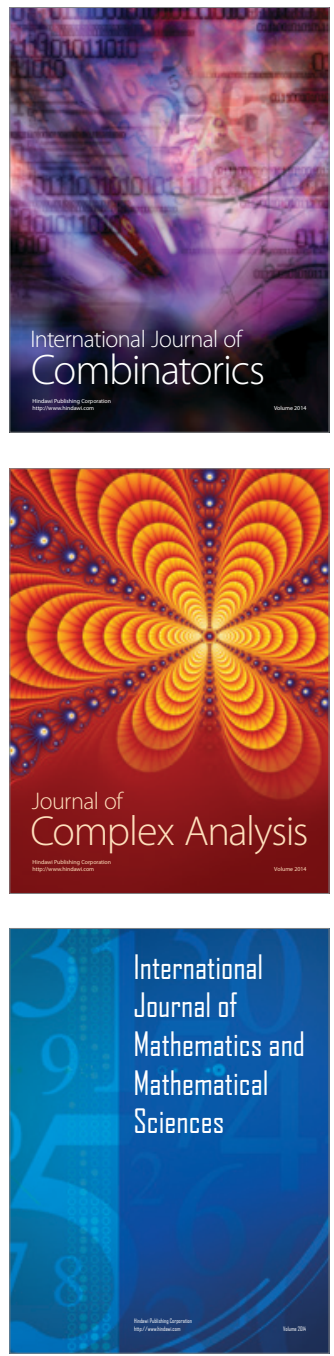
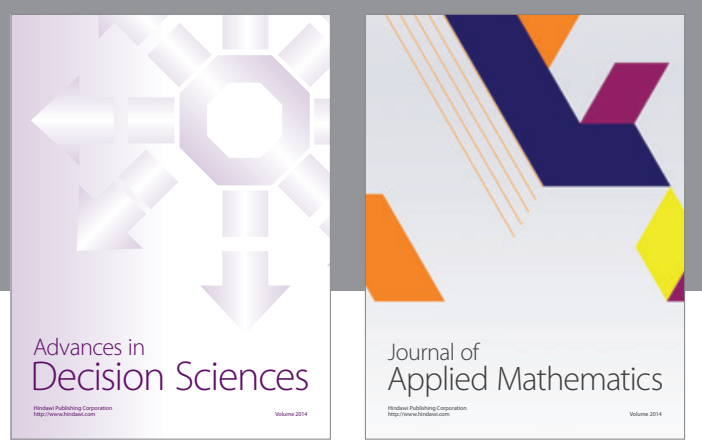

Algebra

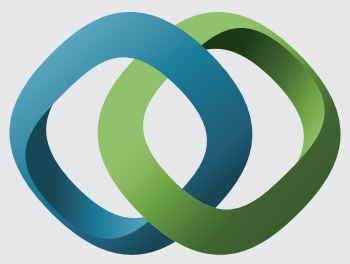

\section{Hindawi}

Submit your manuscripts at

http://www.hindawi.com
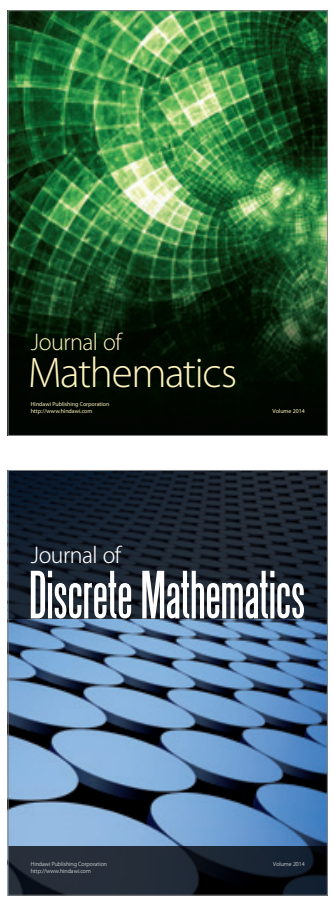

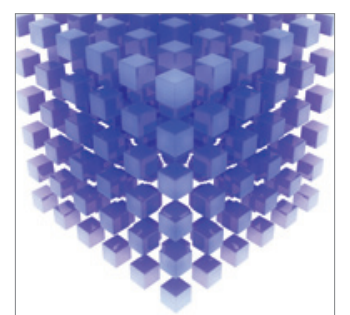

Mathematical Problems in Engineering
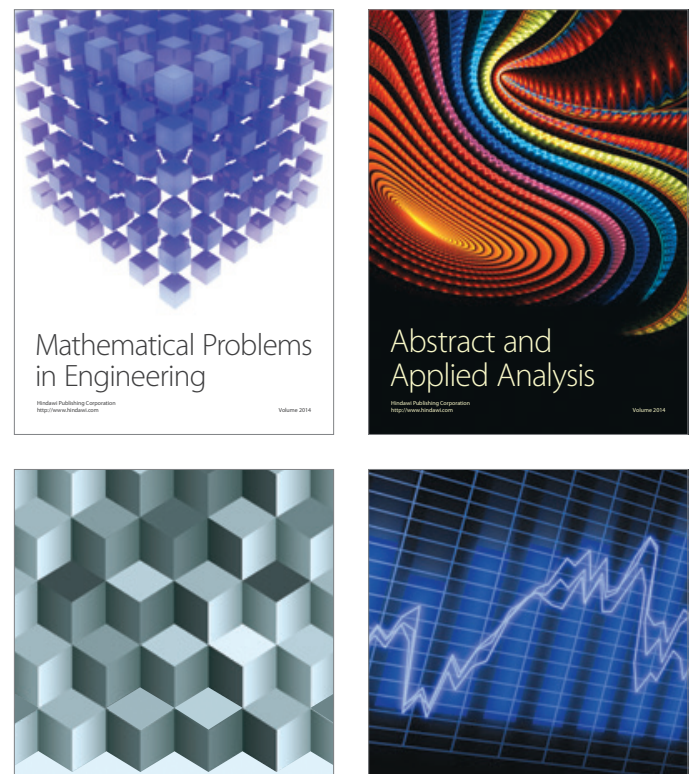

Journal of

Function Spaces

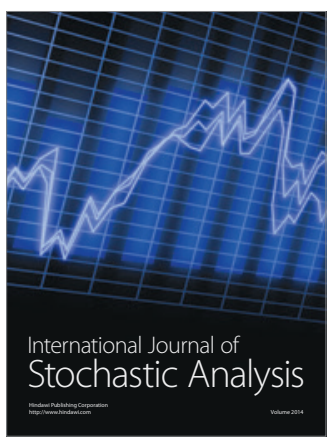

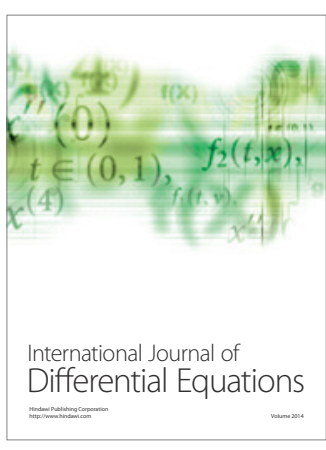
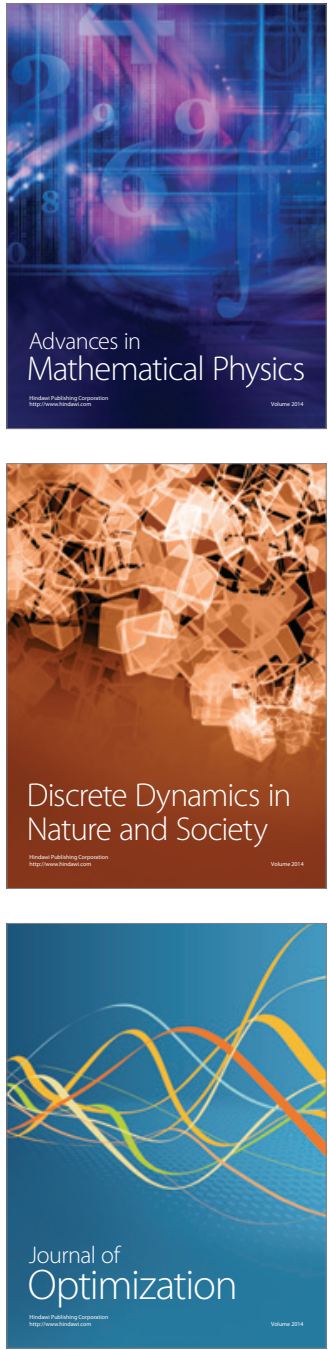